\title{
Applications of quantum stochastic processes in quantum optics
}

\author{
Luc Bouten \\ Physical Measurement and Control 266-33, California Institute of Technology, \\ 1200 E. California Blvd., Pasadena, CA 91125, USA
}

\begin{abstract}
These lecture notes provide an introduction to quantum filtering and its applications in quantum optics. We start with a brief introduction to quantum probability, focusing on the spectral theorem. Then we introduce the conditional expectation and quantum stochastic calculus. In the last part of the notes we discuss the filtering problem.
\end{abstract}

\section{Quantum probability}

In quantum theory observables are represented by selfadjoint operators on a Hilbert space. When an observable is being measured, we randomly obtain a measurement result. That is, the result of the measurement is given by a random variable on some classical probability space. In this section we will investigate how we can pass from a selfadjoint operator to a classical random variable. Along the way we will setup a generalised theory of probability, called noncommutative or quantum probability, that is rich enough to contain quantum mechanics.

\subsection{The spectral theorem}

In these notes we take as a part of its definition that a Hilbert space is separable. The following theorem shows that a self-adjoint operator $S$ on a Hilbert space can be identified with a random variable $h$ on a measure space $(\Omega, \Sigma, \mu)$. It is the heart of quantum mechanics.

Theorem 1.1: (Spectral Theorem) Let $\mathcal{H}$ be a Hilbert space and let $S: \mathcal{H} \rightarrow \mathcal{H}$ be a bounded selfadjoint operator. Then there exist a measure space $(\Omega, \Sigma, \mu)$, a unitary $U: \mathcal{H} \rightarrow L^{2}(\Omega, \Sigma, \mu)$ 
and a bounded real valued $\Sigma$-measurable function $h$ on $\Omega$ such that

$$
S=U^{*} M_{h} U \text {, }
$$

where the multiplication operator $M_{h}: L^{2}(\Omega, \Sigma, \mu) \rightarrow L^{2}(\Omega, \Sigma, \mu)$ is given by

$$
\left(M_{h} f\right)(\omega)=h(\omega) f(\omega),
$$

for all $\omega \in \Omega$.

Proof. See Reed and Simon volume I [23].

Suppose that the dimension $n$ of $\mathcal{H}$ is finite. Since $S$ is self-adjoint, we can always find an orthonormal basis $\left(e_{1}, \ldots, e_{n}\right)$ such that $S$ is diagonal, i.e. $S=\operatorname{diag}\left(h_{1}, \ldots, h_{n}\right)$. Note that the real numbers $h_{i}$ and $h_{j}$ are not necessarily different numbers, some eigenvalues might be degenerate. Let us define $\Omega=\{1, \ldots, n\}$. Moreover, define $h: \Omega \rightarrow \mathbb{R}$ by $h(\omega)=h_{\omega}$ for all $\omega \in \Omega$. Let $\Sigma=\mathcal{P}(\Omega)$ be the sigma-algebra of all subsets of $\Omega$. We take for $\mu$ simply the counting measure. Define $U: \mathcal{H} \rightarrow L^{2}(\Omega, \Sigma, \mu)$ by linear extension of the map

$$
U e_{\omega}=\chi_{\{\omega\}}, \quad \omega \in \Omega,
$$

where $\chi_{\{\omega\}}\left(\omega^{\prime}\right)$ is 1 if $\omega^{\prime}=\omega$ and 0 otherwise. Note that $U$ is unitary and that $S=U^{*} M_{h} U$. That means, we have now proved the spectral theorem in finite dimension. We now also see that the main idea of the theorem is to diagonalise an operator. The spectral theorem gives a precize meaning to the notion of diagonalisation also in the infinite dimensional case.

Let $\mathfrak{X}: \mathbb{R} \rightarrow \mathbb{R}$ be the map given by $\mathfrak{X}(x)=x$ and let $p=\sum_{m=0}^{l} \alpha_{m} \mathfrak{X}^{m}$ be a polynomial. Suppose $S$ is a self-adjoint operator on a Hilbert space $\mathcal{H}$. The spectral theorem provides a measure space $(\Omega, \Sigma, \mu)$, a unitary $U: \mathcal{H} \rightarrow L^{2}(\Omega, \Sigma, \mu)$ and a $\Sigma$-measurable real valued function $h$ such that $S=U^{*} M_{h} U$. It is easy to see that

$$
\sum_{m=0}^{l} \alpha_{m} S^{m}=U^{*} M_{p \circ h} U .
$$

This shows that we can define the polynomial $p$ evaluated at $S$, denoted $p(S)$, in two equivalent ways, i.e. either by the left hand side or the right hand of Eq. (1). The spectrum of an operator $T: \mathcal{H} \rightarrow \mathcal{H}$ is defined as the set

$$
\sigma(T)=\{\lambda \in \mathbb{C} ; T-\lambda I \text { does not have a bounded inverse }\} .
$$

In finite dimensions the spectrum of an operator is just the set of its eigenvalues. The spectrum of a bounded operator $T$ is compact. For the operator $S$, diagonalised by the spectral theorem as $S=U^{*} M_{h} U$, it is not hard to see that $h(\omega) \in \sigma(S)$ for $\mu$-almost all $\omega \in \Omega$ and that $\sigma(S)$ is the smallest compact subset of $\mathbb{C}$ with this property. We denote the range of $h$ by $\operatorname{Ran}(h)$ and are guided by the idea $\operatorname{Ran}(h)=\sigma(S)$.

Definition 1.2: (Function calculus) Let $S: \mathcal{H} \rightarrow \mathcal{H}$ be a bounded, self-adjoint operator and let $j: \sigma(S) \rightarrow \mathbb{C}$ be a bounded Borel measurable function. The spectral theorem provides a measure space $(\Omega, \Sigma, \mu)$, a unitary $U: \mathcal{H} \rightarrow L^{2}(\Omega, \Sigma, \mu)$ and a $\Sigma$-measurable real valued function $h$ such that $S=U^{*} M_{h} U$. Define the bounded linear operator $j(S): \mathcal{H} \rightarrow \mathcal{H}$ by

$$
j(S)=U^{*} M_{j \circ h} U .
$$


For polynomials $p$ it is clear from Eq. (1) that this definition does not depend on which 'diagonalisation' of $S$ we choose (the spectral theorem shows existence, not uniqueness of $(\Omega, \Sigma, \mu), U$ and $h)$. With polynomials we can uniformly approximate continuous functions arbitrarily well $(\sigma(S)$ is compact) and with continuous functions we can approximate bounded Borel measurable functions arbitrarily well pointwise. In this way it follows that the definition of $j(S)$ does not depend on the diagonalisation for all bounded measurable functions $j$. See Reed and Simon [23] for further information.

Suppose the bounded self-adjoint operator $S$ represents an observable of some physical system. Suppose the system is in a state given by a vector $v$ of unit length in the Hilbert space $\mathcal{H}$. We now discuss some of the basics of quantum mechanics in this setting. When it is measured, the observable $S$ can only take values in the spectrum $\sigma(S)$. Let $B$ be a Borel subset of $\sigma(S)$. The set $B$ corresponds to the event ' $S$ takes a value in $B$ '. The probability of this event is given by

$$
\mathbf{P}(S \text { takes a value in } B)=\left\langle v, \chi_{B}(S) v\right\rangle,
$$

where $\chi_{B}$ is the characteristic function of the Borel set $B$, i.e. $\chi_{B}(s)=1$ if $s \in B$ and zero otherwise. The spectral theorem provides a measure space $(\Omega, \Sigma, \mu)$, a unitary $U: \mathcal{H} \rightarrow L^{2}(\Omega, \Sigma, \mu)$ and a $\Sigma$-measurable real valued function $h$ such that $S=U^{*} M_{h} U$. By definition $\chi_{B}(S)=U^{*} M_{\chi_{B} \circ h} U=$ $U^{*} M_{\chi_{h-1(B)}} U$. Note that since $h$ is measurable, the set $h^{-1}(B)$ is an element of $\Sigma$. In fact, we can assign probabilities to all $Q \in \Sigma$ by

$$
\mathbf{P}(Q)=\left\langle v, U^{*} M_{\chi_{Q}} U v\right\rangle .
$$

Summarizing, we can represent $S$ as a random variable $h$ on the probability space $(\Omega, \Sigma, \mathbf{P})$. The spectral theorem transforms quantum mechanics to classical probability theory.

We will now extend the spectral theorem so that we can simultaneously diagonalise a whole class of commuting bounded normal operators. An operator $S: \mathcal{H} \rightarrow \mathcal{H}$ is called normal if it commutes with its adjoint, i.e. $S S^{*}=S^{*} S$. Before stating the theorem, we need to introduce some mathematical objects. Let $\mathcal{H}$ be a Hilbert space. Denote by $\mathcal{B}(\mathcal{H})$ the algebra of all bounded operators on $\mathcal{H}$ and let $\mathcal{S}$ be a subset of $\mathcal{B}(\mathcal{H})$. We call the set

$$
\mathcal{S}^{\prime}=\{R \in \mathcal{B}(\mathcal{H}) ; R S=S R \forall S \in \mathcal{S}\},
$$

the commutant of $\mathcal{S}$ in $\mathcal{B}(\mathcal{H})$. A von Neumann algebra $\mathcal{A}$ on $\mathcal{H}$ is a ${ }^{*}$-subalgebra of $\mathcal{B}(\mathcal{H})$ that equals its double commutant, i.e. $\mathcal{A}^{\prime \prime}=\mathcal{A}$. It is a consequence of von Neumann's double commutant theorem (see e.g. [17]) that a von Neumann algebra is closed in the weak operator topology. It immediately follows from $\mathcal{A}^{\prime \prime}=\mathcal{A}$ that the identity $I \in \mathcal{B}(\mathcal{H})$ is an element of the von Neumann algebra $\mathcal{A}$. A state is a linear map $\rho: \mathcal{A} \rightarrow \mathbb{C}$ such that $\rho$ is positive in the sense that $\rho\left(S^{*} S\right) \geq 0$ for all $S \in \mathcal{A}$, and such that $\rho$ is normalised $\rho(I)=1$. A state is called normal if it is weak operator continuous on the unit ball of $\mathcal{A}$.

Theorem 1.3: (Spectral Theorem) Let $\mathcal{C}$ be a commutative von Neumann algebra and let $\rho$ be a normal state on $\mathcal{C}$. There exist a measure space $(\Omega, \Sigma, \mu)$ and a ${ }^{*}$-isomorphism $\iota$ from $\mathcal{C}$ to $L^{\infty}(\Omega, \Sigma, \mu)$, the space of all bounded measurable functions on $\Omega$. Furthermore, there exists a probability measure $\mathbf{P}$ on $\Sigma$ such that

$$
\rho(C)=\int_{\Omega} \iota(C)(\omega) \mathbf{P}(d \omega),
$$

for all $C \in \mathcal{C}$. 
Theorem 1.3 can be obtained from Theorem 1.1 by using that under mild conditions (which are fulfilled since our von Neumann algebra acts on a separable Hilbert space) a commutative von Neumann algebra $\mathcal{C}$ is generated by a single bounded selfadjoint operator $S$ [27].

Given a probability space $(\Omega, \Sigma, \mathbf{P})$, we can study the commutative von Neumann algebra $\mathcal{C}:=$ $L^{\infty}(\Omega, \Sigma, \mathbf{P})$, acting on the Hilbert space $L^{2}(\Omega, \Sigma, \mathbf{P})$ by pointwise multiplication equipped with the normal state $\rho$ given by expectation with respect to the measure $\mathbf{P}$. The pair $(\mathcal{C}, \rho)$ faithfully encodes the probability space $(\Omega, \Sigma, \mathbf{P})$ [19]. Indeed, the sigma-algebra $\Sigma$ can be reconstructed (up to equivalence of sets with $\mathbf{P}$-null symmetric difference, a point on which we will not dwell here) as the set of projections in $\mathcal{C}$, i.e. the set of characteristic functions of sets in $\Sigma$, and the probability measure is given by acting with the state $\rho$ on this set of projections. We conclude that studying commutative von Neumann algebras equipped with normal states is the same as studying probability spaces. This motivates the following definition.

Definition 1.4: A non-commutative or quantum probability space is a pair $(\mathcal{A}, \rho)$ where $\mathcal{A}$ is a von Neumann algebra on some Hilbert space $\mathcal{H}$ and $\rho$ is a normal state.

Note that we do not require the state $\rho$ to be faithful. We use quantum probability spaces to model experiments involving quantum mechanics. The first thing to do is to set a model, say $(\mathcal{A}, \rho)$. It is a feature of quantum mechanics that in one single realization of an experiment only commuting observables can be measured. That is, the experiment is determined by a commutative von Neumann subalgebra $\mathcal{C}$ of $\mathcal{A}$. The pair $\left(\mathcal{C},\left.\rho\right|_{\mathcal{C}}\right)$ is equivalent to a classical probability model $(\Omega, \Sigma, \mathbf{P})$ via the spectral theorem. The operators in $\mathcal{C}$ are mapped to random variables $\iota(C)$ on $(\Omega, \Sigma, \mathbf{P})$ and represent the stochastic measurement results of the experiment. The pair $(\mathcal{A}, \rho)$ describes the collection of all the experiments (i.e. the collection of commutative subalgebras of $\mathcal{A}$ ) and their statistics in different realizations of the experiment in a concise way.

\subsection{Unbounded operators}

Up to now we have only considered bounded operators. In general, however, an observable is given by a selfadjoint, not necessarily bounded, operator on some dense domain of the Hilbert space $\mathcal{H}$. In these notes we will not put emphasis on the problems one encounters when dealing with unbounded operators. When adding or multiplying unbounded operators, it is important to keep track of the domains involved. In this subsection we discuss some techniques for relating an unbounded operator to a von Neumann algebra.

Definition 1.5: Let $T$ be a densely defined linear operator on some Hilbert space $\mathcal{H}$. Denote the domain of $T$ by $\operatorname{Dom}(T)$. The operator $T$ is called closed if its graph $\{(x, T x) ; x \in \operatorname{Dom}(T)\}$ is a closed subset of $\mathcal{H} \times \mathcal{H}$. Define $\operatorname{Dom}\left(T^{*}\right)$ as the set of $x \in \mathcal{H}$ for which there is a $y \in \mathcal{H}$ such that

$$
\langle T z, x\rangle=\langle z, y\rangle, \quad \forall z \in \operatorname{Dom}(T) .
$$

For each such $x \in \operatorname{Dom}\left(T^{*}\right)$, we define $T^{*} x=y$. We call $T^{*}$ the adjoint of $T$. The operator $T$ is called symmetric if $T \subset T^{*}$, i.e. $\operatorname{Dom}(T) \subset \operatorname{Dom}\left(T^{*}\right)$ and $T z=T^{*} z$ for all $z \in \operatorname{Dom}(T)$. Equivalently, $T$ is symmetric if and only if $\langle T z, y\rangle=\langle z, T y\rangle$ for all $z, y \in \operatorname{Dom}(\mathrm{T})$. The operator $T$ is called selfadjoint if $T=T^{*}$, i.e. if $T$ is symmetric and $\operatorname{Dom}(T)=\operatorname{Dom}\left(T^{*}\right)$. 
Theorem 1.6: (basic criterion for selfadjointness) Let $T$ be a symmetric operator on a Hilbert space $\mathcal{H}$. The following three statements are equivalent

1. $T$ is selfadjoint.

2. $T$ is closed, $\operatorname{Ker}\left(T^{*}+i I\right)=\{0\}$ and $\operatorname{Ker}\left(T^{*}-i I\right)=\{0\}$.

3. $\operatorname{Ran}(T+i I)=\mathcal{H}$ and $\operatorname{Ran}(T-i I)=\mathcal{H}$.

The proof of the above theorem can be found in [23]. Theorem 1.6 shows that $T+i I$ is an injective operator with range $\mathcal{H}$. This means $(T+i I)^{-1}$ is well-defined with domain $\mathcal{H}$. Moreover, since $T+i I$ is closed, $(T+i I)^{-1}$ is also closed. The closed graph theorem (see [23]) then asserts that $(T+i I)^{-1}$ is bounded. A similar argument shows that $T-i I$ is invertible and that $(T-i I)^{-1}$ is bounded. Let $\lambda$ be in the complement of the spectrum of $T$ (i.e. $T-\lambda I$ has a bounded inverse). Define the resolvent of $T$ at $\lambda$ as $R_{\lambda}(T)=(T-\lambda I)^{-1}$. Note that we have the following identity

$$
R_{\lambda}(T)-R_{\mu}(T)=R_{\lambda}(T)(T-\mu I) R_{\mu}(T)-R_{\lambda}(T)(T-\lambda I) R_{\mu}(T)=(\lambda-\mu) R_{\lambda}(T) R_{\mu}(T) .
$$

Adding this same identity with the roles of $\mu$ and $\lambda$ interchanged shows that $R_{\lambda}(T)$ and $R_{\mu}(T)$ commute. Therefore $(T+i I)^{-1}$ and $(T-i I)^{-1}$ commute. The equality

$$
\left\langle(T-i I) x,(T+i I)^{-1}(T+i I) y\right\rangle=\left\langle(T-i I)^{-1}(T-i I) x,(T+i I) y\right\rangle, \quad x, y \in \mathcal{H},
$$

and the fact that $\operatorname{Ran}(T \pm i I)=\mathcal{H}$ shows that $(T+i I)^{-1 *}=(T-i I)^{-1}$. Therefore $(T+i I)^{-1}$ is normal and therefore the von Neumann algebra generated by $(T+i I)^{-1}$ is commutative.

Definition 1.7: Let $\mathcal{A}$ be a von Neumann algebra on some Hilbert space $\mathcal{H}$. Let $T$ be a selfadjoint operator on a dense domain of $\mathcal{H}$. $T$ is said to be affiliated to $\mathcal{A}$ if $(T-i I)^{-1}$ is an element of $\mathcal{A}$. We denote this as $T \eta \mathcal{A}$.

Note that $T$ is affiliated to the commutative von Neumann algebra generated by $(T+i I)^{-1}$. Suppose that $\mathcal{C}$ is a commutative von Neumann algebra on some Hilbert space $\mathcal{H}$ and suppose that $T$ is a selfadjoint operator affiliated to $\mathcal{C}$. Let $\rho$ be a normal state on $\mathcal{C}$. The spectral theorem (Theorem 1.3) provides a measure space $(\Omega, \Sigma, \mu)$, a ${ }^{*}$-isomorphism from $\mathcal{C} \rightarrow L^{\infty}(\Omega, \Sigma, \mu)$ and a probability measure $\mathbf{P}$ on $\Sigma$ such that $\rho(C)=\mathbb{E}_{\mathbf{P}}(\iota(C))$ for all $C \in \mathcal{C}$. That is, $\iota$ allows us to represent the elements in $\mathcal{C}$ as classical random variables on the probability space $(\Omega, \Sigma, \mathbf{P})$. Since $(T+i I)^{-1}$ is an element of $\mathcal{C}$ we can now also represent $T$ on $(\Omega, \Sigma, \mathbb{P})$ by

$$
\iota(T)(\omega)=\frac{1}{\iota\left((T+i I)^{-1}\right)(\omega)}-i, \quad \omega \in \Omega
$$

Note that $\iota(T)$ is not bounded. Nevertheless, we have succeeded in representing $T$ as a classical random variable on $(\Omega, \Sigma, \mathbf{P})$.

The following theorem shows there is a one-one correspondence between strongly continuous oneparameter groups of unitaries and selfadjoint operators. The theorem is a classic result and can be found e.g. in [17] or [23]. 
Theorem 1.8: (Stone's theorem) Let $\mathcal{A}$ be a von Neumann algebra on some Hilbert space $\mathcal{H}$ and let $\left\{U_{t}\right\}_{t \in \mathbb{R}} \subset \mathcal{A}$ be a group of unitaries that is continuous in the strong operator topology. There exists a unique selfadjoint operator $S$ affiliated to $\mathcal{A}$ such that $U_{t}=\exp ($ it $S)$ for all $t \in \mathbb{R}$. The operator $S$ is called the Stone generator of $\left\{U_{t}\right\}_{t \in \mathbb{R}}$.

\subsection{Wiener and Poisson processes}

We now introduce the quantum probability space with which we will model the quantized electromagnetic field. We will see that the model is rich enough to support an entire family of Wiener and Poisson processes. These different processes do not all commute with each other. In this sense quantum probability is richer than the classical theory.

Definition 1.9: Let $\mathcal{H}$ be a Hilbert space. The bosonic or symmetric Fock space over $\mathcal{H}$ is the Hilbert space

$$
\mathcal{F}(\mathcal{H})=\mathbb{C} \oplus \bigoplus_{n=1}^{\infty} \mathcal{H}^{\otimes_{s} n},
$$

where $\otimes_{s}$ denotes the symmetric tensor product.

The Fock space $\mathcal{F}(\mathcal{H})$ describes a field of bosons. The different levels in the Fock space correspond to different numbers of photons present in the field. Note that it is possible to have superpositions between states with different number of particles. For $f \in \mathcal{H}$ we define the exponential vector $e(f)$ by

$$
e(f)=1 \oplus \bigoplus_{n=1}^{\infty} \frac{1}{\sqrt{n !}} f^{\otimes n} .
$$

The vector $\Phi=e(0)=1 \oplus 0 \oplus 0 \oplus \ldots$ is called the vacuum vector. The set of all exponential vectors is linearly independent and the linear span of all exponential vectors $\mathcal{D}$ is a dense subset of $\mathcal{F}(\mathcal{H})$ (see e.g. [21]). On the dense domain $\mathcal{D}$ we define for all $f \in \mathcal{H}$ an operator $W(f)$ by

$$
W(f) e(g)=e^{-\langle f, g\rangle-\frac{1}{2}\|f\|^{2}} e(f+g), \quad g \in \mathcal{H} .
$$

$W(f)$ is an isometric map $\mathcal{D} \rightarrow \mathcal{D}$. Therefore, $W(f)$ extends uniquely to a unitary operator on $\mathcal{F}(\mathcal{H})$. The extension of $W(f)$ to $\mathcal{F}(\mathcal{H})$ is also denoted by $W(f)$. The operators $W(f)$ are called Weyl operators. It can be shown (e.g. [21]) that the Weyl operators $W(f), f \in \mathcal{H}$ generate the algebra $\mathcal{W}=\mathcal{B}(\mathcal{F}(\mathcal{H})$ ) of all bounded operators on $\mathcal{F}(\mathcal{H})$. From the definition in (4) it is easy to see that they satisfy the following Weyl relations

$$
\begin{array}{ll}
\text { 1. } & W(f)^{*}=W(-f), \quad f \in \mathcal{H}, \\
\text { 2. } & W(f) W(g)=e^{-i \operatorname{Im}\langle f, g\rangle} W(f+g), \quad f, g \in \mathcal{H} .
\end{array}
$$

For a fixed $f$ in $\mathcal{H}$, the family $\{W(s f)\}_{s \in \mathbb{R}}$ forms a one-parameter group of unitaries. This oneparameter group is in fact continuous with respect to the strong operator topology [22]. Denote its Stone generator by $B(f)$, i.e. we have $W(s f)=\exp (i s B(f))$. The operators $B(f)$ are called field operators. Let $\mathcal{H}=L^{2}\left(\mathbb{R}^{+}\right)$and fix $\alpha \in[0, \pi)$. It immediately follows from the Weyl relations 
Eq. (5) that all the operators in the set $\mathcal{S}=\left\{W(s f) ; f=e^{i \alpha} \chi_{[0, t]}, t \geq 0, s \in \mathbb{R}\right\}$ commute. Here $\chi_{[0, t]}$ is the indicator function of the interval $[0, t]$, i.e. the function that is 1 on $[0, t]$ and 0 elsewhere. Let $\mathcal{C}^{\alpha}$ be the commutative von Neumann algebra generated by $\mathcal{S}$. Define for all $t \geq 0$ the following selfadjoint operators affiliated to $\mathcal{C}^{\alpha}$

$$
B_{t}^{\alpha}:=B\left(e^{i \alpha} \chi_{[0, t]}\right) .
$$

Let the vacuum state $\phi$ be given by $\phi=\langle\Phi, \cdot \Phi\rangle$. The spectral theorem (Theorem 1.3) provides a measure space $\left(\Omega^{\alpha}, \Sigma^{\alpha}, \mu^{\alpha}\right)$, a ${ }^{*}$-isomorphism $\iota: \mathcal{C}^{\alpha} \rightarrow L^{\infty}\left(\Omega^{\alpha}, \Sigma^{\alpha}, \mu^{\alpha}\right)$, and a probability measure $\mathbf{P}^{\alpha}$ on $\Sigma^{\alpha}$ such that $\phi(C)=\mathbb{E}_{\mathbf{P}^{\alpha}}(\iota(C))$ for all $C \in \mathcal{C}^{\alpha}$. Using the definition in Eq. (3) we can represent the operators $B_{t}^{\alpha}$ for $t \geq 0$ as random variables $\iota\left(B_{t}^{\alpha}\right)$ on $\left(\Omega^{\alpha}, \Sigma^{\alpha}, \mathbf{P}^{\alpha}\right)$. Note that in the above discussion the parameter $\alpha$ was fixed. The operators $B_{t}^{\alpha}$ and $B_{s}^{\alpha^{\prime}}$ for $\alpha \neq \alpha^{\prime}$ do not commute, i.e. they can not be represented as random variables on the same classical probability space.

We will now study the process $\iota\left(B_{t}^{\alpha}\right), t \geq 0$ on $\left(\Omega^{\alpha}, \Sigma^{\alpha}, \mathbf{P}^{\alpha}\right)$. We calculate the characteristic function of an increment $\iota\left(B_{t}^{\alpha}\right)-\iota\left(B_{s}^{\alpha}\right)$ (where $t \geq s$ )

$$
\begin{aligned}
\mathbb{E}_{\mathbf{P} \alpha}\left(e^{i k\left(\iota\left(B_{t}^{\alpha}\right)-\iota\left(B_{s}^{\alpha}\right)\right)}\right) & =\mathbb{E}_{\mathbf{P}^{\alpha}}\left(\iota\left(e^{i k\left(B_{t}^{\alpha}-B_{s}^{\alpha}\right)}\right)\right)=\phi\left(W\left(k \chi_{[s, t]}\right)\right)=\left\langle\Phi, e^{-\frac{1}{2} k^{2}(t-s)} e\left(k \chi_{[s, t]}\right)\right\rangle \\
& =e^{-\frac{1}{2} k^{2}(t-s)}, \quad k \in \mathbb{R} .
\end{aligned}
$$

This shows that the increment has a mean zero Gaussian distribution with variance $t-s$ (see e.g. [28]). Furthermore, a similar calculation shows that the joint characteristic function of two increments is given by (where $t_{1} \geq s_{1} \geq t_{2} \geq s_{2}$ )

$$
\begin{aligned}
& \mathbb{E}_{\mathbf{P}^{\alpha}}\left(e^{i k_{1}\left(\iota\left(B_{t_{1}}^{\alpha}\right)-\iota\left(B_{s_{1}}^{\alpha}\right)\right)+i k_{2}\left(\iota\left(B_{t_{2}}^{\alpha}\right)-\iota\left(B_{s_{2}}^{\alpha}\right)\right)}\right)=e^{-\frac{1}{2}\left(k_{1}^{2}\left(t_{1}-s_{1}\right)+k_{2}^{2}\left(t_{2}-s_{2}\right)\right)}= \\
& \mathbb{E}_{\mathbf{P}^{\alpha}}\left(e^{i k_{1}\left(\iota\left(B_{t_{1}}^{\alpha}\right)-\iota\left(B_{s_{1}}^{\alpha}\right)\right)}\right) \mathbb{E}_{\mathbf{P}^{\alpha}}\left(e^{i k_{2}\left(\iota\left(B_{t_{2}}^{\alpha}\right)-\iota\left(B_{s_{2}}^{\alpha}\right)\right)}\right), \quad k_{1}, k_{2} \in \mathbb{R} .
\end{aligned}
$$

Since the joint characteristic function is multiplicative, the increments are independent [28]. That is, the process $\iota\left(B_{t}^{\alpha}\right)$ has independent mean zero Gaussian increments with variance the length of the increment. This means $\iota\left(B_{t}^{\alpha}\right)$ is a Wiener process. Note that for every $\alpha \in[0, \pi)$ we have now constructed a Wiener process on the Fock space. Note that for different values of $\alpha$ these processes do not commute with each other. The idea to simultaneously diagonalise the fields $\left\{B_{t}^{\alpha}\right\}_{t \geq 0}$ is implicit in some of the earliest work on quantum field theory. However, Segal [24] in the 1950s was the first to emphasize the connection with probability theory.

We will now construct a Poisson process on the Fock space. The second quantization of an element $A \in \mathcal{B}(\mathcal{H})$ such that $\|A\| \leq 1$ (i.e. a contraction) is defined by

$$
\Gamma(A)=I \oplus \bigoplus_{n=1}^{\infty} A^{\otimes n} .
$$

For all contractions $A, B \in \mathcal{B}(\mathcal{H})$ we immediately have $\Gamma(A) \Gamma(B)=\Gamma(A B)$. This means that if $A$ and $B$ commute, then $\Gamma(A)$ and $\Gamma(B)$ also commute. Let $S$ be a selfadjoint element of $\mathcal{B}(\mathcal{H})$. The selfadjoint operator $S$ generates a one-parameter group $U_{s}=\exp ($ is $S)$ of unitaries in $\mathcal{B}(\mathcal{H})$. After second quantization this leads to a one-parameter group $\Gamma(\exp ($ is $S))$ of unitaries in $\mathcal{W}=\mathcal{B}(\mathcal{F}(\mathcal{H}))$. Denote the Stone generator of $\Gamma(\exp (i t S))$ by $\Lambda(S)$, i.e. $\Gamma(\exp (i s S))=\exp (i s \Lambda(S))$. Note that $\Lambda(S)$ is affiliated to the commutative von Neumann algebra generated by $\left\{\Gamma\left(U_{s}\right)\right\}_{s \in \mathbb{R}}$. 
Let $\mathcal{H}=L^{2}\left(\mathbb{R}^{+}\right)$. For $t \geq 0$, let $P_{t}$ be the projection given by $P_{t}=M_{\chi_{[0, t]}}$. $P_{t}$ is selfadjoint and therefore generates a one-parameter group $U_{s}^{t}=\exp \left(i s P_{t}\right)$. Let $\mathcal{C}$ be the commutative algebra generated by all $U_{s}^{t}$ for $s \in \mathbb{R}, t \geq 0$. Introduce the shorthand $\Lambda_{t}$ for the Stone generators $\Lambda\left(P_{t}\right)$. Note that for all $t \geq 0, \Lambda_{t}$ is affiliated to $\mathcal{C}$. On the $n$th layer of the symmetric Fock space $\Gamma\left(\exp \left(i s P_{t}\right)\right)$ acts as $\exp \left(i s P_{t}\right)^{\otimes n}$. Differentiation with respect to $s$ shows that on the $n$th layer of the symmetric Fock space $\Lambda_{t}=P_{t} \otimes I^{n-1}+I \otimes P_{t} \otimes I^{n-2}+\ldots+I^{n-1} \otimes P_{t}$. This shows that $\Lambda_{t}$ counts how many particles (i.e. photons) are present in the interval $[0, t]$.

For $f \in L^{2}(\mathbb{R})$ we define a coherent vector $\psi(f)$ as the exponential vector normalised to unit length, i.e.

$$
\psi(f)=e^{-\frac{1}{2}\|f\|^{2}} e(f)=W(f) e(0)=W(f) \Phi .
$$

We can now define a coherent state on $\mathcal{W}=\mathcal{B}\left(\mathcal{F}\left(L^{2}\left(\mathbb{R}^{+}\right)\right)\right)$by $\rho(A)=\langle\psi(f), A \psi(f)\rangle$. The spectral theorem (Theorem 1.3) provides a measure space $(\Omega, \Sigma, \mu)$, a ${ }^{*}$-isomorphism $\iota: \mathcal{C} \rightarrow L^{\infty}(\Omega, \Sigma, \mu)$ and a probability measure $\mathbf{P}$ such that $\mathbb{E}_{\mathbf{P}}(\iota(C))=\rho(C)$ for all $C \in \mathcal{C}$. Since the selfadjoint operators $\Lambda_{t}$ are affiliated with $\mathcal{C}$, we can represent them as random variables $\iota\left(\Lambda_{t}\right)$ on $(\Omega, \Sigma, \mathbf{P})$.

For $t_{1} \geq s_{1} \geq t_{2} \geq s_{2}$ the joint characteristic function of the increments $\iota\left(\Lambda_{t_{1}}\right)-\iota\left(\Lambda_{s_{1}}\right)$ and $\iota\left(\Lambda_{t_{2}}\right)-\iota\left(\Lambda_{s_{2}}\right)$ is given by

$$
\begin{aligned}
& \mathbb{E}_{\mathbf{P}}\left(e^{i k_{1}\left(\iota\left(\Lambda_{t_{1}}\right)-\iota\left(\Lambda_{s_{1}}\right)\right)+i k_{2}\left(\iota\left(\Lambda_{t_{2}}\right)-\iota\left(\Lambda_{s_{2}}\right)\right)}\right)= \\
& \left\langle\psi(f), \Gamma\left(e^{i k_{1}\left(P_{t_{1}}-P_{s_{1}}\right)}\right) \Gamma\left(e^{i k_{2}\left(P_{t_{2}}-P_{s_{2}}\right)}\right) \psi(f)\right\rangle=e^{-\|f\|^{2}}\left\langle e(f), e\left(e^{i k_{1}\left(P_{t_{1}}-P_{s_{1}}\right)} e^{i k_{2}\left(P_{t_{2}}-P_{s_{2}}\right)} f\right)\right\rangle= \\
& e^{\left\langle f,\left(e^{i k_{1}\left(P_{t_{1}}-P_{s_{1}}\right)} e^{i k_{2}\left(P_{t_{2}}-P_{s_{2}}\right)}-1\right) f\right\rangle}=e^{\int_{s_{1}}^{t_{1}}\left(e^{i k_{1}}-1\right)|f|^{2} d \lambda} e^{\int_{s_{2}}^{t_{2}}\left(e^{i k_{2}}-1\right)|f|^{2} d \lambda}, \quad k_{1}, k_{2} \in \mathbb{R} .
\end{aligned}
$$

This shows that $\left\{\iota\left(\Lambda_{t}\right)\right\}_{t \geq 0}$ is a process with independent increments. Moreover, it shows that $\iota\left(\Lambda_{t}\right)_{t \geq 0}$ is a Poisson process with intensity measure $|f|^{2} d \lambda$, where $\lambda$ stands for the Lebesgue measure. Since $\psi(f)=W(f) \Phi$, we can also work with respect to the vacuum by sandwiching $\Lambda_{t}$ by $W(f)$. We conclude that in the vacuum the process $W(f)^{*} \Lambda_{t} W(f)$ is a Poisson process with intensity measure $|f|^{2} d \lambda$.

\section{Conditional expectations}

In this section we start with a discussion to illustrate how the classical conditional expectation can be transferred to quantum models using the spectral theorem. In pushing the classical concept as far as possible, we arrive at a definition for the quantum conditional expectation. In Section 4 we state the quantum filtering problem in terms of the newly defined quantum conditional expectation.

\subsection{Towards a definition}

Let $\mathcal{B}$ be a von Neumann algebra on a Hilbert space $\mathcal{H}$ and let $\mathbb{P}$ be a normal state on $\mathcal{B}$. Let $X$ and $Y$ be two selfadjoint commuting elements of $\mathcal{B}$. Their expectations are given by $\mathbb{P}(X)$ and 
$\mathbb{P}(Y)$, respectively. In this subsection we show how to define the conditional expectation $\mathbb{P}(X \mid Y)$ of $X$ given $Y$.

Let us first recall the classical definition. Suppose that $F$ and $G$ are random variables on a probability space $(\Omega, \Sigma, \mathbf{P})$. Let us suppose for convenience that $\Omega$ is a finite set. Since $G$ is a function on a finite set, its range $\operatorname{Ran}(G)$ is also a finite set. The classical conditional expectation $\mathbb{E}_{\mathbf{P}}(F \mid G)$ of $F$ given $G$ is the random variable (i.e. a measurable function from $\Omega$ to $\mathbb{C}$ ) given by

$$
\mathbb{E}_{\mathbf{P}}(F \mid G)(\omega)=\sum_{g \in \operatorname{Ran}(G)} \frac{\mathbb{E}_{\mathbf{P}}\left(F \chi_{[G=g]}\right)}{\mathbf{P}([G=g])} \chi_{[G=g]}(\omega), \quad \omega \in \Omega,
$$

where $[G=g]$ is the set $\{\omega \in \Omega ; G(w)=g\}$ and $\chi_{[G=g]}$ is the indicator function of that set. Note that $\mathbb{E}_{\mathbf{P}}(F \mid G)$ is not just $\Sigma$-measurable, but even $\sigma(G)$-measurable, where $\sigma(G)$ is the $\sigma$-algebra generated by $G$. If $\Omega$ is not a finite set, and might even be continuous, this last point is the guiding idea. The conditional expectation is then defined as the orthogonal projection from $L^{2}(\Omega, \Sigma, \mathbf{P})$ onto $L^{2}\left(\Omega, \sigma(G), \mathbf{P}\right.$ ) (and then extended to $L^{1}$ ), see [28].

Instead of a direct definition as in Eq. (7), or as an orthogonal projection on an $L^{2}$-space, we can provide an abstract characterisation of the conditional expectation. Given a $\sigma$-subalgebra $\Sigma_{0}$ of $\Sigma$, we call a $\Sigma_{0}$-measurable random variable $\mathbb{E}_{\mathbf{P}}\left(F \mid \Sigma_{0}\right)$ a version of the conditional expectation of $F$ on $\Sigma_{0}$, if for all $\Sigma_{0}$-measurable random variables $S$ we have

$$
\mathbb{E}_{\mathbf{P}}\left(\mathbb{E}_{\mathbf{P}}\left(F \mid \Sigma_{0}\right) S\right)=\mathbb{E}_{\mathbf{P}}(F S) .
$$

In geometric terms this is just a characterisation of the projection discussed above. Therefore, there exists an object that satisfies this definition, see [28]. It is easy to see that the direct definition in Eq. (7) satisfies the abstract characterisation of Eq. (8) if we take $\Sigma_{0}=\sigma(G)$. Furthermore, there is almost surely only one $\mathbb{E}_{\mathbf{P}}\left(F \mid \Sigma_{0}\right)$ satisfying (8) (hence the terminology "...a version of the..."). The proof of this statement is the same as that for the quantum case, which we will give below. Starting from the definition of the classical conditional expectation $\mathbb{E}_{\mathbf{P}}\left(F \mid \Sigma_{0}\right)$ in Eq. (8) we can prove that it has the following properties see e.g. [28]. It is linear in $F$, it maps positive random variables to positive random variables, it preserves $\chi_{\Omega}$, it has the module property $\mathbb{E}_{\mathbf{P}}\left(F S \mid \Sigma_{0}\right)=$ $\mathbb{E}_{\mathbf{P}}\left(F \mid \Sigma_{0}\right) S$ for all $\Sigma_{0}$-measurable functions $S$, it satisfies the tower property $\mathbb{E}_{\mathbf{P}}\left(\mathbb{E}_{\mathbf{P}}\left(F \mid \Sigma_{1}\right) \mid \Sigma_{0}\right)=$ $\mathbb{E}_{\mathbf{P}}\left(F \mid \Sigma_{0}\right)$ whenever $\Sigma_{0} \subset \Sigma_{1}$, and it is the least mean square estimate of $F$ (see also further below).

Let us return to the commuting selfadjoint operators $X$ and $Y$ in the von Neumann algebra $\mathcal{B}$ equipped with the normal state $\mathbb{P}$. The operators $X$ and $Y$ together generate a commutative von Neumann subalgebra $\mathcal{X}$ of $\mathcal{B}$. The spectral theorem provides a measure space $(\Omega, \Sigma, \mu)$, a * isomorphism $\iota$ from $\mathcal{X}$ to $L^{\infty}(\Omega, \Sigma, \mu)$ and a probability measure $\mathbf{P}$ on $\Sigma$ such that $\mathbb{P}(C)=\mathbb{E}_{\mathbf{P}}(\iota(C))$ for all $C \in \mathcal{X}$. We know how to condition $\iota(X)$ on $\iota(Y)$, that can be done with the classical conditional expectation $\mathbb{E}_{\mathbf{P}}(\iota(X) \mid \iota(Y))$. It is natural to define the quantum conditional expectation as

$$
\mathbb{P}(X \mid Y)=\iota^{-1}\left(\mathbb{E}_{\mathbf{P}}(\iota(X) \mid \iota(Y))\right) .
$$

We now see that we can only condition those observables $X \in \mathcal{B}$ that commute with $Y$. That, however, is exactly as it should be. In one realization of an experiment we can only access commuting observables. Two noncommuting observables can never both be assigned a numerical value in a single realization of the experiment, i.e. there is no need for conditioning them on each other. 
This idea has been called the nondemolition principle. Note, however, that two operators $X_{1}$ and $X_{2}$ that both commute with $Y$ need not necessarily commute with each other.

It is now straightforward to define the conditional expectation of a selfadjoint operator $X$ on a commutative subalgebra $\mathcal{C}$ of $\mathcal{B}$ with which $X$ commutes, i.e. $X C=C X$ for all $C \in \mathcal{C}$. In short, let $\mathcal{X}$ be the commutative algebra generated by the whole of $\mathcal{C}$ and $X$ together. Apply the spectral theorem to $(\mathcal{X}, \mathbb{P})$, that enables the definition of a $\iota$, and then define

$$
\mathbb{P}(X \mid \mathcal{C}):=\iota^{-1}\left(\mathbb{E}_{\mathbf{P}}(\iota(X) \mid \sigma(\iota(C) ; C \in \mathcal{C}))\right)
$$

In the next subsection we start with the formal definition of the quantum conditional expectation.

\subsection{The quantum conditional expectation}

Definition 2.1: (Quantum conditional expectation) Let $(\mathcal{B}, \mathbb{P})$ be a quantum probability space. Let $\mathcal{C}$ be a commutative von Neumann subalgebra of $\mathcal{B}$. Denote by $\mathcal{A}$ its relative commutant, i.e. $\mathcal{A}=\mathcal{C}^{\prime}:=\{A \in \mathcal{B} ; A C=C A, \forall C \in \mathcal{C}\}$. Then $\mathbb{P}(\cdot \mid \mathcal{C}): \mathcal{A} \rightarrow \mathcal{C}$ is (a version of) the conditional expectation from $\mathcal{A}$ onto $\mathcal{C}$ if

$$
\mathbb{P}(\mathbb{P}(A \mid \mathcal{C}) C)=\mathbb{P}(A C), \quad \forall A \in \mathcal{A}, \forall C \in \mathcal{C} .
$$

Note that the conditional expectation $\mathbb{P}(\cdot \mid \mathcal{C})$ is defined only on the commutant $\mathcal{A}$ of $\mathcal{C}$ ! The definition that we gave here is more restrictive than the one that is usual in quantum probability (see e.g. [26]). There, one also allows for the conditioning on noncommutative subalgebras.

In applications, we start with the quantum probability space $(\mathcal{B} \otimes \mathcal{W}, \mathbb{P})$. Here $\mathcal{B}$ is the algebra with which we model some system of interest (two-level atom, a gas of atoms etc.), $\mathcal{W}=\mathcal{B}\left(\mathcal{F}\left(L^{2}(\mathbb{R})\right)\right)$ is the algebra with which we model the electromagnetic field and $\mathbb{P}=\rho \otimes \phi$, where $\rho$ some normal state on $\mathcal{B}$ and $\phi$ is the vacuum state on the field. A commutative subalgebra $\mathcal{C}$ is then generated by the observations we perform (see further below). Next, we choose $\mathcal{A}$ to be the relative commutant of $\mathcal{C}$. The algebra $\mathcal{A}$ consists of the observables that have not been demolished by our observations, i.e. it consists of all operators that are still compatible with $\mathcal{C}$. The following lemma establishes existence and uniqueness for the conditional expectation of Definition 2.1.

Lemma 2.2: The conditional expectation of Definition 2.1 exists and is unique with probability one, i.e. any two versions $P$ and $Q$ of $\mathbb{P}(A \mid \mathcal{C})$ satisfy $\|P-Q\|_{\mathbb{P}}=0$, where $\|X\|_{\mathbb{P}}^{2}:=\mathbb{P}\left(X^{*} X\right)$.

Proof. Existence. For a self-adjoint element $A \in \mathcal{A}$ we can define $\mathbb{P}(A \mid \mathcal{C})$ via Eq. (9). We now need to check that it satisfies the abstract characterisation Eq. (10). That, however, follows easily from the classical couterpart Eq. (8). If $A \in \mathcal{A}$ is not self-adjoint, then we write it as the sum of two self-adjoint elements

$$
A=\frac{\left(A+A^{*}\right)-i\left(i\left(A-A^{*}\right)\right)}{2}
$$


and simply extend $\mathbb{P}(\cdot \mid \mathcal{C})$ linearly. It is easy to see that that obeys Eq. (10).

Uniqueness w.p. one. Define the pre-inner product $\langle X, Y\rangle:=\mathbb{P}\left(X^{*} Y\right)$ on $\mathcal{A}$ (it might have nontrivial kernel if $\mathbb{P}$ is not faithful.) Then $\langle C, A-\mathbb{P}(A \mid \mathcal{C})\rangle=\mathbb{P}\left(C^{*} A\right)-\mathbb{P}\left(C^{*} \mathbb{P}(A \mid \mathcal{C})\right)=0$ for all $C \in \mathcal{C}$ and $A \in \mathcal{A}$, i.e. $A-\mathbb{P}(A \mid \mathcal{C})$ is orthogonal to $\mathcal{C}$. Now let $P$ and $Q$ be two versions of $\mathbb{P}(A \mid \mathcal{C})$. It follows that $\langle C, P-Q\rangle=0$ for all $C \in \mathcal{C}$. But $P-Q \in \mathcal{C}$, so $\langle P-Q, P-Q\rangle=\|P-Q\|_{\mathbb{P}}^{2}=0$.

The next lemma asserts that, as in the classical case, the conditional expectation is the least mean square estimate.

Lemma 2.3: $\mathbb{P}(A \mid \mathcal{C})$ is the least mean square estimate of $A$ given $\mathcal{C}$, i.e.

$$
\|A-\mathbb{P}(A \mid \mathcal{C})\|_{\mathbb{P}} \leq\|A-K\|_{\mathbb{P}}, \quad \forall K \in \mathcal{C} .
$$

Proof. For all $K \in \mathcal{C}$ we have

$$
\begin{aligned}
\|A-K\|_{\mathbb{P}}^{2} & =\|A-\mathbb{P}(A \mid \mathcal{C})+\mathbb{P}(A \mid \mathcal{C})-K\|_{\mathbb{P}}^{2} \\
& =\|A-\mathbb{P}(A \mid \mathcal{C})\|_{\mathbb{P}}^{2}+\|\mathbb{P}(A \mid \mathcal{C})-K\|_{\mathbb{P}}^{2} \geq\|A-\mathbb{P}(A \mid \mathcal{C})\|_{\mathbb{P}}^{2},
\end{aligned}
$$

where, in the next to last step, we used that $A-\mathbb{P}(A \mid \mathcal{C})$ is orthogonal to $\mathbb{P}(A \mid \mathcal{C})-K \in \mathcal{C}$.

Remark. We have now highlighted the existence, uniqueness with probability one, and the mean least squares property of the quantum conditional expectation. The other elementary properties of classical conditional expectations and their proofs [28] carry over directly to the noncommutative setting. In particular, we have linearity, positivity, preservation of the identity, the tower property $\mathbb{P}(\mathbb{P}(A \mid \mathcal{B}) \mid \mathcal{C})=\mathbb{P}(A \mid \mathcal{C})$ if $\mathcal{C} \subset \mathcal{B}$, the module property $\mathbb{P}(A B \mid \mathcal{C})=\mathbb{P}(A \mid \mathcal{C}) B$ for $B \in \mathcal{C}$, etc. As an example, let us prove linearity. It suffices to show that $Z=\alpha \mathbb{P}(A \mid \mathcal{C})+\beta \mathbb{P}(B \mid \mathcal{C})$ satisfies the definition of $\mathbb{P}(\alpha A+\beta B \mid \mathcal{C})$, i.e. $\mathbb{P}(Z C)=\mathbb{P}((\alpha A+\beta B) C)$ for all $C \in \mathcal{C}$. But this is immediate from the linearity of $\mathbb{P}$ and Definition 2.1 .

In Section 4 we will need to relate conditional expectations with respect to different states to each other. This is done by the following Bayes-type formula.

Lemma 2.4: (Bayes formula $[8][7])$ Let $(\mathcal{B}, \mathbb{P})$ be a noncommutative probability space. Let $\mathcal{C}$ be a commutative von Neumann subalgebra of $\mathcal{B}$ and let $\mathcal{A}$ be its relative commutant, i.e. $\mathcal{A}=\mathcal{C}^{\prime}:=\{A \in \mathcal{B} ; A C=C A, \forall C \in \mathcal{C}\}$. Furthermore, let $Q$ be an element in $\mathcal{A}$ such that $Q^{*} Q$ is invertible and $\mathbb{P}\left(Q^{*} Q\right)=1$. We can define a state on $\mathcal{A}$ by $\mathbb{Q}(A):=\mathbb{P}\left(Q^{*} A Q\right)$ and we have

$$
\mathbb{Q}(X \mid \mathcal{C})=\frac{\mathbb{P}\left(Q^{*} X Q \mid \mathcal{C}\right)}{\mathbb{P}\left(Q^{*} Q \mid \mathcal{C}\right)}, \quad X \in \mathcal{A}
$$


Proof. Let $K$ be an element of $\mathcal{C}$. For all $X \in \mathcal{A}$, we can write

$$
\begin{aligned}
& \mathbb{P}\left(\mathbb{P}\left(Q^{*} X Q \mid \mathcal{C}\right) K\right)=\mathbb{P}\left(Q^{*} X K Q\right)=\mathbb{Q}(X K)=\mathbb{Q}(\mathbb{Q}(X \mid \mathcal{C}) K)= \\
& \mathbb{P}\left(Q^{*} Q \mathbb{Q}(X \mid \mathcal{C}) K\right)=\mathbb{P}\left(\mathbb{P}\left(Q^{*} Q \mathbb{Q}(X \mid \mathcal{C}) K \mid \mathcal{C}\right)\right)= \\
& \mathbb{P}\left(\mathbb{P}\left(Q^{*} Q \mid \mathcal{C}\right) \mathbb{Q}(X \mid \mathcal{C}) K\right) .
\end{aligned}
$$

Note that the invertibility of $\mathbb{P}\left(Q^{*} Q \mid \mathcal{C}\right)$ follows immediately from the invertibility of $Q^{*} Q$.

\section{Quantum stochastic calculus}

We have seen in Section 1.3 that the quantum probability space $(\mathcal{W}, \phi)$ contains a rich variety of Wiener and Poisson processes. We will now introduce stochastic integrals with respect to these processes and study their stochastic calculus. Quantum stochastic calculus has been introduced by Hudson and Parthasarathy [16]. Quantum stochastic differential equations describe the interaction between atoms and the electromagnetic field in the weak coupling limit.

\subsection{The stochastic integral}

Lemma 3.1: Let $\mathcal{H}_{1}$ and $\mathcal{H}_{2}$ be Hilbert spaces. There exists a unique unitary isomorphism $U: \mathcal{F}\left(\mathcal{H}_{1} \oplus \mathcal{H}_{2}\right) \rightarrow \mathcal{F}\left(\mathcal{H}_{1}\right) \otimes \mathcal{F}\left(\mathcal{H}_{2}\right)$ such that

$$
U e(x \oplus y)=e(x) \otimes e(y), \quad x \in \mathcal{H}_{1}, y \in \mathcal{H}_{2} .
$$

Proof. By linear extension of the relation in Eq. (11) we get a surjective map from $\mathcal{D}=\operatorname{span}\{e(x) ; x \in$ $\left.\mathcal{H}_{1} \oplus \mathcal{H}_{2}\right\}$ to $\mathcal{D}_{1} \otimes \mathcal{D}_{2}$ where $\mathcal{D}_{i}=\operatorname{span}\left\{e(x) ; x \in \mathcal{H}_{i}\right\}$. Note that $\mathcal{D}$ is dense in $\mathcal{F}\left(\mathcal{H}_{1} \oplus \mathcal{H}_{2}\right)$ and $\mathcal{D}_{1} \otimes \mathcal{D}_{2}$ is dense in $\mathcal{F}\left(\mathcal{H}_{1}\right) \otimes \mathcal{F}\left(\mathcal{H}_{2}\right)$. Moreover, for all $x_{1}, y_{1} \in \mathcal{H}_{1}$ and $x_{2}, y_{2} \in \mathcal{H}_{2}$ we have

$$
\left\langle e\left(x_{1} \oplus x_{2}\right), e\left(y_{1} \oplus y_{2}\right)\right\rangle=e^{\left\langle x_{1}+x_{2}, y_{1}+y_{2}\right\rangle}=e^{\left\langle x_{1}, y_{1}\right\rangle} e^{\left\langle x_{2}, y_{2}\right\rangle}=\left\langle e\left(x_{1}\right), e\left(y_{1}\right)\right\rangle\left\langle e\left(x_{2}\right), e\left(y_{2}\right)\right\rangle,
$$

i.e. $U: \mathcal{D} \rightarrow \mathcal{D}_{1} \otimes \mathcal{D}_{2}$ is isometric and therefore extends uniquely to a unitary.

We will often identify $\mathcal{F}\left(\mathcal{H}_{1} \oplus \mathcal{H}_{2}\right)$ and $\mathcal{F}\left(\mathcal{H}_{1}\right) \otimes \mathcal{F}\left(\mathcal{H}_{2}\right)$ using the unitary of Lemma 3.1. For all $t \geq$ $s \geq 0$ we define $\mathcal{F}=\mathcal{F}\left(L^{2}\left(\mathbb{R}^{+}\right)\right), \mathcal{F}_{t]}=\mathcal{F}\left(L^{2}([0, t])\right), \mathcal{F}_{[s, t]}=\mathcal{F}\left(L^{2}([s, t])\right)$ and $\mathcal{F}_{[t}=\mathcal{F}\left(L^{2}([t, \infty))\right.$. For $t_{n}>\ldots>t_{1}>0$ we have $L^{2}\left(\mathbb{R}^{+}\right)=L^{2}\left(\left[0, t_{1}\right]\right) \oplus L^{2}\left(\left[t_{1}, t_{2}\right]\right) \oplus \ldots \oplus L^{2}\left(\left[t_{n}, \infty\right)\right)$. Therefore, in the sense of Lemma 3.1, we get

$$
\mathcal{F}=\mathcal{F}_{\left.t_{1}\right]} \otimes \mathcal{F}_{\left[t_{1}, t_{2}\right]} \otimes \ldots \otimes \mathcal{F}_{\left[t_{n-1}, t_{n}\right]} \otimes \mathcal{F}_{\left[t_{n}\right.}
$$

Every partition leads to a tensor product splitting of the symmetric Fock space. In this sense the symmetric Fock space is a continuous tensor product. Let $\mathcal{H}_{1}$ and $\mathcal{H}_{2}$ be Hilbert spaces. Denote $\mathcal{H}=\mathcal{H}_{1} \oplus \mathcal{H}_{2}$. It easily follows from the definition Eq. (4) that

$$
W\left(x_{1} \oplus x_{2}\right)=W\left(x_{1}\right) \otimes W\left(x_{2}\right), \quad x_{1} \in \mathcal{H}_{1}, x_{2} \in \mathcal{H}_{2}
$$


This means that the algebra generated by the Weyl operators also splits as a continuous tensor product, i.e. for $t_{n}>\ldots>t_{1}>0$ we have

$$
\mathcal{W}=\mathcal{W}_{\left.t_{1}\right]} \otimes \mathcal{W}_{\left[t_{1}, t_{2}\right]} \otimes \ldots \otimes \mathcal{W}_{\left[t_{n-1}, t_{n}\right]} \otimes \mathcal{W}_{[t}
$$

where $\mathcal{W}=\mathcal{B}\left(L^{2}([0, \infty))\right), \mathcal{W}_{t]}=\mathcal{B}\left(L^{2}([0, t])\right), \mathcal{W}_{[s, t]}=\mathcal{B}\left(L^{2}([s, t])\right)$ and $\mathcal{W}_{[t}=\mathcal{B}\left(L^{2}([t, \infty))\right)$. Furthermore, from the definition in Eq. (6) it follows that

$$
\Gamma\left(S_{1} \oplus S_{2}\right)=\Gamma\left(S_{1}\right) \otimes \Gamma\left(S_{2}\right)
$$

for all contractions $S_{1} \in \mathcal{B}\left(\mathcal{H}_{1}\right)$ and $S_{2} \in \mathcal{B}\left(\mathcal{H}_{2}\right)$.

Definition 3.2: On the dense domain $\mathcal{D}=\operatorname{span}\left\{e(f) ; f \in L^{2}\left(\mathbb{R}^{+}\right)\right\}$we introduce annihilation $A_{t}$ and creation $A_{t}$ operators by

$$
A_{t}=\frac{1}{2}\left(B\left(i \chi_{[0, t]}\right)-i B\left(\chi_{[0, t]}\right)\right), \quad A_{t}^{*}=\frac{1}{2}\left(B\left(i \chi_{[0, t]}\right)+i B\left(\chi_{[0, t]}\right)\right),
$$

where $B(f)$ denotes the Stone generator of $W(t f)$. The restriction of $\Lambda_{t}$ to $\mathcal{D}$ is also denoted by $\Lambda_{t}$ and is called the gauge process. The operators $A_{t}, A_{t}^{*}$ and $\Lambda_{t}$ are called the fundamental noises.

It can be shown e.g. [21] that the domain of the Stone generators $B(f), f \in L^{2}\left(\mathbb{R}^{+}\right)$contains $\mathcal{D}$, that $A_{t} e(f)=\left\langle\chi_{[0, t]}, f\right\rangle e(f)$ and that $A_{t}^{*}$ is the adjoint of $A_{t}$ restricted to $\mathcal{D}$. Moreover, from its definition it follows that $\left\langle e(f), \Lambda_{t} e(f)\right\rangle=\left\langle g, \chi_{[0, t]} f\right\rangle\langle e(g), e(f)\rangle$. In particular this means that $A_{t} \Phi=A_{t} e(0)=0$ and $\Lambda_{t} \Phi=0$, properties that we will exploit later on. Let $M_{t}$ be one of the fundamental noises. It is a consequence of Eqs. (12) and (13) and the definition of the fundamental noises as (linear combinations of) generators of one-parameter groups that

$$
\left(M_{t}-M_{s}\right) e(f)=e\left(f_{s]}\right) \otimes\left(\left(M_{t}-M_{s}\right) e\left(f_{[s, t]}\right)\right) \otimes e\left(f_{[t}\right),
$$

where $f \in L^{2}\left(\mathbb{R}^{+}\right), f_{s]}=\chi_{[0, s]} f, f_{[s, t]}=\chi_{[0, t]} f, f_{[t}=\chi_{[t, \infty)} f$ and $\left(M_{t}-M_{s}\right) e\left(f_{[s, t]}\right) \in \mathcal{F}_{[s, t]}$. In the following we will for notational convenience often omit the tensor product signs between exponential vectors. Let $\mathcal{H}$ be a Hilbert space, called the initial space. We tensor the initial space to $\mathcal{F}$ and extend the operators $A_{t}, A_{t}^{*}$ and $\Lambda_{t}$ to $\mathcal{H} \otimes \mathcal{F}$ by ampliation, i.e. by tensoring the identity to them on $\mathcal{H}$ (however, to keep notation light we will not denote it). We denote the algebra of all bounded operators on $\mathcal{H}$ by $\mathcal{B}$.

Definition 3.3: (Simple quantum stochastic integral) Let $\left\{L_{s}\right\}_{0 \leq s \leq t}$ be an adapted (i.e. $L_{s} \in \mathcal{B} \otimes \mathcal{W}_{s]}$ for all $\left.0 \leq s \leq t\right)$ simple process with respect to the partition $\left\{s_{0}=0, s_{1}, \ldots, s_{p}=t\right\}$ in the sense that $L_{s}=L_{s_{j}}$ whenever $s_{j} \leq s<s_{j+1}$. The stochastic integral of $L$ with respect to a fundamental noise $M$ on $\mathcal{H} \otimes \mathcal{D}$ is then defined as $[16,21]$

$$
\int_{0}^{t} L_{s} d M_{s} x e(f):=\sum_{j=0}^{p-1}\left(L_{s_{j}} x e\left(f_{\left.s_{j}\right]}\right)\right)\left(\left(M_{s_{j+1}}-M_{s_{j}}\right) e\left(f_{\left[s_{j}, s_{j+1}\right]}\right)\right) e\left(f_{\left[s_{j+1}\right.}\right), \quad x \in \mathcal{H} .
$$

Let $\left\{L_{s}\right\}_{0 \leq s \leq t}$ be an adapted process, i.e. $L_{s} \in \mathcal{B} \otimes \mathcal{W}_{s]}$ for all $0 \leq s \leq t$. We would like to define the integral

$$
I_{t}=\int_{0}^{t} L_{s} d M_{s}
$$


as a limit of simple integrals $I_{t}^{n}=\int_{0}^{t} L_{s}^{n} d M_{s}$ where $L^{n}$ is an approximation of $L$ by simple adapted processes. In the classical case we use the Itô isometry to define the stochastic integral as a mean square limit of simple processes. Moreover, one can show that each mean square integrable process can be approximated by simple processes. Let us see how far we would get using this procedure in the quantum case. For simplicity suppose that the initial space is trivial $\mathcal{H}=\mathbb{C}$. Suppose that we are working with respect to the vacuum state $\phi$ on $\mathcal{W}$. Following the classical reasoning, we are looking for an operator $I_{t}$ such that $\left\langle\left(I_{t}-I_{t}^{n}\right) \Phi,\left(I_{t}-I_{t}^{n}\right) \Phi\right\rangle \rightarrow 0$ as $n \rightarrow \infty$ where $I_{t}^{n}$ are simple integrals corresponding to simple approximations of $L$. This however, would only fix the action of $I_{t}$ on the vacuum vector $\Phi$. It remains unclear what the domain of $I_{t}$ is and what the action of $I_{t}$ is on the vectors in that domain that are not the vacuum.

The solution to this problem was given by Hudson and Parthasarathy [16]. They simply fix the domain for a stochastic integral $I_{t}$ to $\mathcal{H} \otimes \mathcal{D}$ (one could choose a dense domain in $\mathcal{H}$, for simplicity we have chosen $\mathcal{H})$. Moreover, they consider $I_{t}^{n}$ to be an approximation of $I_{t}$ if $\left\langle\left(I_{t}-I_{t}^{n}\right) x \otimes\right.$ $\left.\psi,\left(I_{t}-I_{t}^{n}\right) x \otimes \psi\right\rangle \rightarrow 0$ as $n \rightarrow \infty$ for all $x \in \mathcal{H}$ and $\psi \in \mathcal{D}$. This limit exists if $\int_{0}^{t}\left\|L_{s}-L_{s}^{n}\right\| d s \rightarrow 0$ as $n \rightarrow \infty$ for all $x \in \mathcal{H}, \psi \in \mathcal{D}$ and the limit is independent of the approximation [16]. Moreover, every adapted square integrable process $L$, i.e. $\int_{0}^{t}\left\|L_{s} x \otimes \psi\right\|^{2} d s<\infty$ for all $x \in \mathcal{H}, \psi \in \mathcal{D}$ admits a simple approximation [16]. This means that for every adapted square integrable $L_{s}$ we now have an unambiguous definition of the stochastic integral $\int_{0}^{t} L_{s} d M_{s}$, where $M_{s}$ can be any of the three fundamental noises. Adapted square integrable processes are said to be stochastically integrable. We use the following shorthand for stochastic integrals $d X_{t}=L_{t} d M_{t}$ means $X_{t}=X_{0}+\int_{0}^{t} L_{s} d M_{s}$.

Since $A_{t} \Phi=\Lambda_{t} \Phi=0$ it is immediate from the definition that quantum stochastic integrals with respect to $A_{t}$ and $\Lambda_{t}$ acting on $\Phi$ are zero, or infinitesimally $d \Lambda_{t} \Phi_{[t}=d A_{t} \Phi_{[t}=0$. From this we can immediately conclude that vacuum expectations of stochastic integrals with respect to $A_{t}$ and $\Lambda_{t}$ vanish. Furthermore, we have $\left\langle\Phi, \int_{0}^{t} L_{s} d A_{s}^{*} \Phi\right\rangle=\left\langle\int_{0}^{t} L_{s}^{*} d A_{s} \Phi, \Phi\right\rangle=0$, i.e. vacuum expectation of stochastic integrals with respect to $A_{t}^{*}$ are zero as well. Note, however, that $d A_{t}^{*} \Phi_{[t} \neq 0$.

To get some more feeling for the definition of the quantum stochastic integral we will now investigate which quantum stochastic differential equation is satisfied by the Weyl operators $W\left(f_{t]}\right)$ $\left(f \in L^{2}\left(\mathbb{R}^{+}\right)\right)$. Note that the stochastic integrals are defined on the domain $\mathcal{D}$. Therefore we calculate for $g$ and $h$ in $L^{2}\left(\mathbb{R}^{+}\right)$

$$
\phi(t):=\left\langle e(g), W\left(f_{t]}\right) e(h)\right\rangle=e^{\left.-\left\langle f_{t]}, h\right\rangle-\frac{1}{2} \| f_{t}\right] \|^{2}}\left\langle e(g), e\left(h+f_{t]}\right)\right\rangle=e^{\left.\left\langle g, f_{t]}\right\rangle-\left\langle f_{t]}, h\right\rangle-\frac{1}{2} \| f_{t}\right] \|^{2}} e^{\langle g, h\rangle},
$$

which means that

$$
\phi(t)-\phi(0)=\int_{0}^{t}\left\langle e(g), \frac{d}{d s}\left(\left\langle g, f_{s]}\right\rangle-\left\langle f_{s]}, h\right\rangle-\frac{1}{2}\left\|f_{s]}\right\|^{2}\right) \phi(s) e(h)\right\rangle d s .
$$

Let us turn to the definition of the stochastic integral, Definition 3.3. Let $\left\{0=s_{0}, s_{1}, \ldots, s_{p}=t\right\}$ be a partition of $[0, t]$ and choose $L_{s}=\bar{f}\left(s_{j}\right) W\left(f_{s_{j}}\right)$ for $s_{j} \leq s<s_{j+1}$. Let further $M_{t}$ be $A_{t}$, then 
the definition of the stochastic integral gives (heuristically in the last step)

$$
\begin{aligned}
& \sum_{j=0}^{p-1}\left(\bar{f}\left(s_{j}\right) W\left(f_{\left.s_{j}\right]}\right) e\left(h_{\left.s_{j}\right]}\right)\right)\left(\left(A_{s_{j+1}}-A_{s_{j}}\right) e\left(h_{\left[s_{j}, s_{j+1}\right]}\right)\right) e\left(h_{\left[s_{j+1}\right.}\right)= \\
& \sum_{j=0}^{p-1}\left\langle f\left(s_{j}\right) \chi_{\left[s_{j}, s_{j+1}\right]}, h\right\rangle W\left(f_{\left.s_{j}\right]}\right) e(h)=\sum_{j=0}^{p-1}\left(\left\langle f\left(s_{j}\right) \chi_{\left.s_{j+1}\right]}, h\right\rangle-\left\langle f\left(s_{j}\right) \chi_{\left.s_{j}\right]}, h\right\rangle\right) W\left(f_{\left.s_{j}\right]}\right) e(h) \\
& \longrightarrow \int_{0}^{t} d\left\langle f_{s]}, h\right\rangle W\left(f_{s]}\right) e(h) .
\end{aligned}
$$

Together with a similar calculation for $M_{t}=A_{t}^{*}$, this yields the following quantum stochastic differential equation for the Weyl operator $W\left(f_{t]}\right)$

$$
d W\left(f_{t]}\right)=\left\{f(t) d A_{t}^{*}-\bar{f}(t) d A_{t}-\frac{1}{2}|f(t)|^{2} d t\right\} W\left(f_{t]}\right) .
$$

\subsection{The calculus}

We now turn to the calculus satisfied by the quantum stochastic integrals we defined in the previous subsection. It is the calculus that makes the theory useful, it allows us to forget the tedious definition of the integral in computations and instead to perform algebraic manipulations with increments.

Definition 3.4: A pair $\left(L, L^{\dagger}\right)$ of adapted processes defined on $\mathcal{H} \otimes \mathcal{D}$ is called an adjoint pair if

$$
\left\langle x \otimes e(f), L_{t} y \otimes e(f)\right\rangle=\left\langle L_{t}^{\dagger} x \otimes e(f), y \otimes e(f)\right\rangle, \quad x, y \in \mathcal{H}, f, g \in L^{2}\left(\mathbb{R}^{+}\right), t \geq 0 .
$$

The dagger replaces the adjoint on the domain $\mathcal{H} \otimes \mathcal{D}$. It is easy to see that $\left(A, A^{*}\right)$ and $(\Lambda, \Lambda)$ are adjoint pairs. Moreover, if $\left(L, L^{\dagger}\right)$ is an adjoint pair, then $\left(X, X^{\dagger}\right)$ is an adjoint pair, where $d X_{t}=L d M_{t}$ and $d X_{t}^{\dagger}=L^{\dagger} d M_{t}^{\dagger}$.

Theorem 3.5: (Quantum Itô rule [16]) Let $\left(B, B^{\dagger}\right),\left(C, C^{\dagger}\right),\left(D, D^{\dagger}\right),\left(E, E^{\dagger}\right)$ be adjoint pairs of stochastically integrable processes. Let $F_{t}, G_{t}, H_{t}$ and $I_{t}$ be stochastically integrable processes. Let $X_{t}$ and $Y_{t}$ be stochastic integrals of the form

$$
\begin{aligned}
& d X_{t}=B_{t} d \Lambda_{t}+C_{t} d A_{t}+D_{t} d A_{t}^{*}+E_{t} d t, \\
& d Y_{t}=F_{t} d \Lambda_{t}+G_{t} d A_{t}+H_{t} d A_{t}^{*}+I_{t} d t,
\end{aligned}
$$

Suppose $X_{t} Y_{t}$ is an adapted process defined on $\mathcal{H} \otimes \mathcal{D}$ and $X F, X G, X H, X I, B Y, C Y, D Y, E Y, B F$, $C F, B H$ and $C H$ are stochastically integrable, then

$$
d\left(X_{t} Y_{t}\right)=X_{t} d Y_{t}+\left(d X_{t}\right) Y_{t}+d X_{t} d Y_{t},
$$

where $d X_{t} d Y_{t}$ should be evaluated according to the quantum Itô table 


\begin{tabular}{l|llll} 
& $d A_{t}$ & $d \Lambda_{t}$ & $d A_{t}^{*}$ & $d t$ \\
\hline$d A_{t}$ & 0 & $d A_{t}$ & $d t$ & 0 \\
$d \Lambda_{t}$ & 0 & $d \Lambda_{t}$ & $d A_{t}^{*}$ & 0 \\
$d A_{t}^{*}$ & 0 & 0 & 0 & 0 \\
$d t$ & 0 & 0 & 0 & 0
\end{tabular}

i.e. $d X_{t} d Y_{t}=B_{t} F_{t} d \Lambda_{t}+C_{t} F_{t} d A_{t}+B_{t} H_{t} d A_{t}^{*}+C_{t} H_{t} d t$.

Suppose that the product $X_{t} Y_{t}$ is an adapted process defined on $\mathcal{H} \otimes \mathcal{D}$. Then we can read off an expression for $X_{t} Y_{t}$ from the matrix elements $\left\langle X_{t}^{\dagger} x \otimes e(f), Y_{t} y \otimes e(g)\right\rangle$, which explains the need for the concept of an adjoint pair. Writing out these matrix elements using the definition of the stochastic integral is the basic ingredient of the proof of the quantum Itô rule, see [16] and [21]. To get some more feeling for the proof of the quantum Itô rule we compute for $x, y \in \mathcal{H}$ and $f, g \in L^{2}\left(\mathbb{R}^{+}\right)$

$$
\begin{aligned}
& \left\langle x e(f), A_{t} A_{t}^{*} y e(g)\right\rangle=\left\langle x e(f),\left(A_{t}^{*} A_{t}+\left[A_{t}, A_{t}^{*}\right]\right) y e(g)\right\rangle= \\
& \left\langle A_{t} x e(f), A_{t} y e(g)\right\rangle+t\langle x e(f), y e(g)\rangle=\left(\left\langle f, \chi_{[0, t]}\right\rangle\left\langle\chi_{[0, t]}, g\right\rangle+t\right)\langle x e(f), y e(g)\rangle .
\end{aligned}
$$

Infinitesimally that reads $d\left(A_{t} A_{t}^{*}\right)=A_{t} d A_{t}^{*}+A_{t}^{*} d A_{t}+d t$. Note that the Itô correction term finds its origins in the commutator between $A_{t}$ and $A_{t}^{*}$.

Note that we need to check many conditions before we are allowed to apply the quantum Itô rule. In practice though we mostly work with 'noisy Schrödinger equations', which have unitary and therefore bounded solutions. If the integrals and coefficients are bounded, then all the requirements of the theorem are satisfied. In the remainder of these notes we will not worry much about these issues and just assume that we can apply the quantum Itô rule.

In Section 1 we encountered the classical Wiener processes $B_{t}^{\alpha}=i e^{-i \alpha} A_{t}-i e^{i \alpha} A_{t}^{*}$ for $\alpha \in[0, \pi)$. Since $d B_{t}^{\alpha}=i e^{-i \alpha} d A_{t}-i e^{i \alpha} d A_{t}^{*}$, we recover the classical Itô rule for these Wiener processes, i.e. $\left(d B_{t}^{\alpha}\right)^{2}=d t$, from the quantum Itô rule. For an $f \in L^{2}\left(\mathbb{R}^{+}\right)$we can write the Weyl operator $W\left(f_{t]}\right)$ as $W\left(f_{t]}\right)=\exp \left(\int_{0}^{t} f(s) d\left(A_{s}^{*}-A_{s}\right)\right)$. Therefore it follows from the quantum Itô rule that the Weyl operators $W\left(f_{t]}\right)$ satisfy equation (15), where $-\frac{1}{2}\|f\|^{2} W\left(f_{t]}\right) d t$ is the Itô correction term. The Poisson process of the previous section was given by $\Lambda_{t}^{f}:=W(f)^{*} \Lambda_{t} W(f)$ in the vacuum state, for which the quantum Itô rule gives

$$
d \Lambda_{t}^{f}=d \Lambda_{t}+\bar{f}(t) d A_{t}+f(t) d A_{t}^{*}+|f(t)|^{2} d t,
$$

which leads to the classical Itô rule $\left(d \Lambda_{t}^{f}\right)^{2}=d \Lambda_{t}^{f}$ for the Poisson process. Furthermore, integrating the above equation, we see that $\Lambda_{t}^{f}=\Lambda_{t}+B\left(i f_{t]}\right)+\int_{0}^{t}|f(s)|^{2} d s$.

\subsection{Open quantum systems}

In quantum optics the basic model consists of some physical system of interest, e.g. a two-level atom, a cloud of atoms, or an atom in a cavity, in interaction with the electromagnetic field. The 
interaction between the electromagnetic field and the system of interest is described by quantum electrodynamics, see e.g. [10]. The dynamics is given by a Schrödinger equation in which the atomic dipole operator couples to a stationary Gaussian wide band noise. The next step is to approximate the wide band noise by white noise. See [1], [14] and [11] for rigorous limits implementing this Markovian approximation. In this procedure the Schrödinger equation given by quantum electrodynamics transforms to a quantum stochastic differential equation of the form

$$
d U_{t}=\left\{(S-I) d \Lambda_{t}+L d A_{t}^{*}-L^{*} S d A_{t}-\frac{1}{2} L^{*} L d t-i H d t\right\} U_{t}, \quad U_{0}=I
$$

where $S, L$ and $H$ are operators on the system of interest, $H$ being selfadjoint and $S$ being unitary. See [14] for a description of how gauge terms can arise from QED Hamiltonians in a Markovian approximation. For simplicity we have considered only one channel in the field. In the examples below we will briefly describe systems that interact with two field channels.

A Picard iteration scheme shows that there exists a unique solution to Eq. (16). Using the quantum Itô rule we can calculate $d U_{t}^{*} U_{t}=d U_{t} U_{t}^{*}=0$, i.e. the solution to Eq. (16) is unitary. We define the time evolution of observables of the system of interest by the flow $j_{t}(X)=U_{t}^{*}(X \otimes I) U_{t}$. Using the quantum Itô rule, we find

$$
d j_{t}(X)=j_{t}(\mathcal{L}(X)) d t+j_{t}\left(S^{*} X S-X\right) d \Lambda_{t}+j_{t}\left(\left[L^{*}, X S\right]\right) d A_{t}+j_{t}\left(\left[S^{*} X, L\right]\right) d A_{t}^{*},
$$

where the Lindblad generator [18] is given by

$$
\mathcal{L}(X)=i[H, X]+L^{*} X L-\frac{1}{2}\left(L^{*} L X-X L^{*} L\right) .
$$

Instead of going through a rigorous Markov limit, we will always take the quantum stochastic differential equation (16) as our starting point.

Example 3.6: Suppose we are studying a two-level atom in interaction with the electromagnetic field. The two-level atom is described by the quantum probability space $\left(M_{2}(\mathbb{C}), \rho\right)$ and the field is described by $(\mathcal{W}, \phi)$. The operators $S, L$ and $H$ are given by

$$
S=I, \quad L=\gamma \sigma_{-}=\left(\begin{array}{ll}
0 & 0 \\
\gamma & 0
\end{array}\right), \quad H=\frac{\hbar \omega_{0}}{2} \sigma_{z}=\left(\begin{array}{cc}
\frac{\hbar \omega_{0}}{2} & 0 \\
0 & -\frac{\hbar \omega_{0}}{2}
\end{array}\right),
$$

where $\hbar$ is Planck's constant, $\gamma \geq 0$ is a decay parameter, and $\omega_{0} \in \mathbb{R}$ is the so-called atomic frequency, determined by the fact that $\hbar \omega_{0}$ is the energy difference between the two levels. That is, the interaction between the two-level atom and the electromagnetic field is given by the following quantum stochastic differential equation $\left(\sigma_{+}=\sigma_{-}^{*}\right)$

$$
d U_{t}=\left\{\gamma \sigma_{-} d A_{t}^{*}-\gamma \sigma_{+} d A_{t}-\frac{\gamma^{2}}{2} \sigma_{+} \sigma_{-} d t-i \frac{\hbar}{2} \sigma_{z}\right\}, \quad U_{0}=I
$$

A laser driving the two-level atom could be modelled by an additional Hamiltonian $\Omega \sigma_{x}=\Omega\left(\sigma_{-}+\right.$ $\left.\sigma_{+}\right)$, with $\Omega \in \mathbb{R}$. A more realistic model is obtained by adding an extra field channel in a coherent state (the laser). If we distinguish two field channels, then the two-level atom and the field together are described by the quantum probability space $\left(M_{2}(\mathbb{C}) \otimes \mathcal{W}_{1} \otimes \mathcal{W}_{2}, \rho \otimes \phi \otimes \sigma\right)$ where $\sigma$ is the state given by the inner product with the vector $\psi(f)=W(f) e(0)$, for some $f \in L^{2}\left(\mathbb{R}^{+}\right)$. Here 
$|f|$ is the amplitude of the laser and the phase of $f$ represents the phase of the driving laser. The quantum stochastic differential equation is given by

$$
\begin{aligned}
& d U_{t}=\left\{\gamma_{1} \sigma_{-} d A_{1 t}^{*}-\gamma_{1} \sigma_{+} d A_{1 t}+\gamma_{2} \sigma_{-} d A_{2 t}^{*}-\gamma_{2} \sigma_{+} d A_{2 t}-\frac{\gamma_{1}^{2}+\gamma_{2}^{2}}{2} \sigma_{+} \sigma_{-} d t-i \frac{\hbar}{2} \sigma_{z}\right\} U_{t}, \\
& U_{0}=I,
\end{aligned}
$$

with $\gamma_{1}, \gamma_{2} \geq 0$. If we are only interested in the evolution of adapted observables, then we have $W(f)^{*} U_{t}^{*} X U_{t} W(f)=W\left(f_{t]}\right)^{*} U_{t}^{*} X U_{t} W\left(f_{t]}\right)$. Therefore we can describe the system by the quantum probability space $\left(M_{2}(\mathbb{C}) \otimes \mathcal{W}_{1} \otimes \mathcal{W}_{2}, \rho \otimes \phi \otimes \phi\right)$, and interaction given by $\tilde{U}_{t}=U_{t} W\left(f_{t]}\right)$. Using the quantum Itô rule it is easy to see that

$$
\begin{aligned}
d \tilde{U}_{t}=\{ & \left\{\gamma_{1} \sigma_{-} d A_{1 t}^{*}-\gamma_{1} \sigma_{+} d A_{1 t}+L d A_{2 t}^{*}-L^{*} d A_{2 t}-\right. \\
& \left.\frac{\gamma_{1}^{2} \sigma_{+} \sigma_{-}+L^{*} L}{2} d t-i \frac{\hbar}{2} \sigma_{z}-i H d t\right\} \tilde{U}_{t}, \quad \tilde{U}_{0}=I,
\end{aligned}
$$

with $L=\gamma_{2} \sigma_{-}+f(t)$ and $H=i\left(\gamma_{2} f(t) \sigma_{+}-\gamma_{2} \overline{f(t)} \sigma_{-}\right) / 2$. The Hamiltonian $H$ reduces to the simpler form $\Omega \sigma_{x}$ if $\Omega=i \gamma_{2} f(t) / 2$ and $f(t)=-i$.

Example 3.7: We consider a two-level atom inside a cavity. The cavity has one leaky mirror which couples it to the outside field. The cavity is described by the Hilbert space $\ell^{2}(\mathbb{N})$. Let $n \in \mathbb{N}$. Denote by $\delta_{n}$ the element of $\ell^{2}(\mathbb{N})$ given by $\delta_{n}(n)=1$ and $\delta_{n}(m)=0$ for all $m \in$ $\mathbb{N} \backslash\{n\}$. The annihilation operator is given by $b \delta_{n}=\sqrt{n} \delta_{n-1}, n \in \mathbb{N}^{*}$ and $b \delta_{0}=0$ (we will not worry about defining a domain for $b$ ). The creation operator $b^{*}$ is the adjoint of $b$. The twolevel atom in the cavity coupled to the field can be described by the quantum probability space $\left(M_{2}(\mathbb{C}) \otimes \mathcal{B}\left(\ell^{2}(\mathbb{N})\right) \otimes \mathcal{W}, \rho \otimes \sigma \otimes \phi\right)$, where $\rho$ is some state of the two-level atom, $\sigma$ is some state on $\mathcal{B}\left(\ell^{2}(\mathbb{N})\right)$ (the cavity) and $\phi$ is the vacuum state of the field. The quantum stochastic differential equation describing the two-level atom, the cavity, and the field together is

$$
d U_{t}=\left\{\gamma b d A_{t}^{*}-\gamma b^{*} d A_{t}-\frac{\gamma^{2}}{2} b^{*} b d t+g\left(\sigma_{+} b-\sigma_{-} b^{*}\right) d t-i\left(\Delta_{a} \sigma_{z}+\Delta_{c} b^{*} b\right) d t\right\} U_{t}, \quad U_{0}=I .
$$

Here $\gamma$ and $g$ are positive parameters, determined by the cavity. The parameters $\Delta_{a}$ and $\Delta_{c}$ are real. More information about cavity QED can be found in [10]. See [13] for rigorous results on systems including a cavity.

Example 3.8: We consider a gas of atoms in free space in interaction with a linearly polarized laser beam [25]. Suppose the atoms are all two-level atoms, i.e. the Hilbert space of the gas is given by $\mathcal{H}:=\mathbb{C}^{2^{N}}$. Here $N$ represents the number of atoms in the gas. The gas is described by the quantum probability space $(\mathcal{B}(\mathcal{H}), \rho)$, where $\rho$ is a state on the algebra $\mathcal{B}(\mathcal{H})$ of all bounded operators on $\mathcal{H}$. We model the laser beam by the symmetric Fock space over the one photon space $\mathbb{C}^{2} \otimes L^{2}\left(\mathbb{R}^{+}\right)$. Here $\mathbb{C}^{2}$ describes the polarization and $L^{2}\left(\mathbb{R}^{+}\right)$describes the spatial degree of freedom of a photon in the beam. Since the speed of light is constant, we can identify the spatial degree of freedom with time. Let $\left\{e_{x}, e_{y}\right\}$ be the orthonormal basis in $\mathbb{C}^{2}$ such that $e_{x}$ corresponds to an $x$-polarized photon and $e_{y}$ to a $y$-polarized photon. We can identify $\mathbb{C}^{2} \otimes L^{2}\left(\mathbb{R}^{+}\right)$with $L^{2}\left(\mathbb{R}^{+}\right) \oplus L^{2}\left(\mathbb{R}^{+}\right)$by the linear map that maps $e_{x} \otimes f$ to $(f, 0)$ and $e_{y} \otimes g$ to $(0, g)$ for all $f, g \in L^{2}\left(\mathbb{R}^{+}\right)$. That is, the laser beam consists out of two polarization channels

$$
\mathcal{F}\left(\mathbb{C}^{2} \otimes L^{2}\left(\mathbb{R}^{+}\right)\right)=\mathcal{F}_{x} \otimes \mathcal{F}_{y} .
$$


Let $\mathcal{W}_{x}$ and $\mathcal{W}_{y}$ be the algebras of all bounded operators on $\mathcal{F}_{x}$ and $\mathcal{F}_{y}$, respectively. The gas and the $x$-polarized laser beam together are modelled by the quantum probability space $(\mathcal{B}(\mathcal{H}) \otimes$ $\left.\mathcal{W}_{x} \otimes \mathcal{W}_{y}, \rho \otimes \sigma\right)$ where $\sigma$ is given by the inner product with the coherent vector $\psi\left(e_{x} \otimes f\right)$ in $\mathcal{F}\left(\mathbb{C}^{2} \otimes L^{2}\left(\mathbb{R}^{+}\right)\right)$given by

$$
\psi\left(e_{x} \otimes f\right)=\exp \left(-\frac{1}{2}\|f\|^{2}\right) 1 \oplus \bigoplus_{n=1}^{\infty} \frac{\left(e_{x} \otimes f\right)^{\otimes n}}{\sqrt{n !}} .
$$

The modulus and phase of $f(t)$ correspond to the amplitude and phase of the laser at time $t$.

We can also choose the circularly polarized basis $\left\{e_{+}, e_{-}\right\}$in $\mathbb{C}^{2}$, given by $e_{+}=-\left(e_{x}+i e_{y}\right) / \sqrt{2}$ and $e_{-}=\left(e_{x}-i e_{y}\right) / \sqrt{2}$. In an analogous way as for the linear basis $\left\{e_{x}, e_{y}\right\}$ this leads to an identification

$$
\mathcal{F}\left(\mathbb{C}^{2} \otimes L^{2}\left(\mathbb{R}^{+}\right)\right)=\mathcal{F}_{+} \otimes \mathcal{F}_{-} .
$$

In a suitable appproximation [25], the interaction between the laser beam and the gas of atoms can be described by the following quantum stochastic differential equation [5]

$$
d U_{t}=\left\{\left(e^{i \kappa F_{z}}-I\right) d \Lambda_{t}^{++}+\left(e^{-i \kappa F_{z}}-I\right) d \Lambda_{t}^{--}\right\} U_{t}, \quad U_{0}=I
$$

Here $\kappa$ is a real coupling parameter. The processes $\Lambda_{t}^{++}$and $\Lambda_{t}^{--}$are the gauge processes on $\mathcal{F}_{+}$ and $\mathcal{F}_{-}$, respectively. The operator $F_{z}: \mathbb{C}^{2^{N}} \rightarrow \mathbb{C}^{2^{N}}$ is the collective $z$-component of the spin of the atoms

$$
F_{z}=\sum_{i=1}^{N} I_{2}^{\otimes i-1} \otimes \sigma_{z} \otimes I_{2}^{\otimes N-i}
$$

Equation (17) shows that right polarized photons rotate the spin over a positive angle $\kappa$ along the $z$-axis and left polarized photons rotate the spin over a negative angle $\kappa$ along the $z$-axis.

\section{Quantum filtering}

In this section we formulate the filtering problem and solve it by a change of measure technique that is inspired by classical filtering theory [20, 12, 29]. Quantum filtering has been introduced by Belavkin using martingale methods [3, 4], see also [6]. The treatment below follows [8, 9] and is based on the quantum Bayes formula and a trick introduced by Holevo [15].

\subsection{The filtering problem}

Let $(\mathcal{B}, \rho)$ be the quantum probability space with which we model a certain system of interest, e.g. a two-level atom, a dilute gas of atoms, an atom in a cavity, or a harmonic oscillator. The system of interest is coupled to the electromagnetic field $(\mathcal{W}, \phi)$ which we will take to be in the vacuum state. The combined system is then described by the quantum probability space $(\mathcal{B} \otimes \mathcal{W}, \mathbb{P})$ where $\mathbb{P}=\rho \otimes \phi$. Suppose the interaction between the system of interest and the electromagnetic field is given by a QSDE of the following type

$$
d U_{t}=\left\{L d A_{t}^{*}-L^{*} d A_{t}-\frac{1}{2} L^{*} L d t-i H d t\right\} U_{t}, \quad U_{0}=I
$$


where $L$ and $H$ are elements in $\mathcal{B}, H$ being selfadjoint. For reasons of convenience we have restricted ourselves to only a single channel in the field and no gauge terms in the QSDE.

We will work in the Heisenberg picture, i.e. the state $\mathbb{P}$ remains fixed and the observables evolve in time according to $j_{t}(S)=U_{t}^{*} S U_{t}$ where $S$ is an element in $\mathcal{B} \otimes \mathcal{W}$. Let $X$ be a system operator, i.e. $X \in \mathcal{B}$, then it follows from the quantum Itô rule that

$$
d j_{t}(X)=j_{t}(\mathcal{L}(X)) d t+j_{t}\left(\left[L^{*}, X\right]\right) d A_{t}+j_{t}([X, L]) d A_{t}^{*}
$$

We will call Eq. (19) the system.

In the field we are doing a measurement continuously in time. We will allow for two possible measurement setups, direct photon detection, for which the observations are given by $Y_{t}^{\Lambda}=U_{t}^{*} \Lambda_{t} U_{t}$, and homodyne detection, for which the observations are given by $Y_{t}^{W}=U_{t}^{*}\left(e^{-i \phi_{t}} A_{t}+e^{i \phi_{t}} A_{t}^{*}\right) U_{t}$. For convenience we will choose $\phi_{t}=0$. We will not go further into the details of the homodyne detection setup here, but instead refer to [2]. Using the quantum Itô rule we obtain

$$
\begin{aligned}
& d Y_{t}^{\Lambda}=d \Lambda_{t}+j_{t}(L) d A_{t}^{*}+j_{t}\left(L^{*}\right) d A_{t}+j_{t}\left(L^{*} L\right) d t \\
& d Y_{t}^{W}=j_{t}\left(L+L^{*}\right) d t+d A_{t}+d A_{t}^{*} .
\end{aligned}
$$

The quantum probability space $(\mathcal{B} \otimes \mathcal{W}, \mathbb{P})$ together with Eqs. (19) and $(20)$ define a systemobservations model, in analogy to the system-observation models in classical stochastic control theory.

Only if the observations at different times commute with each other, can we observe them in a single realization of an experiment. The requirement that the observations are commutative is called the self-nondemolition property. Note that if the observations are self-nondemolition, then they can be mapped onto a classical stochastic process via the spectral theorem. This classical stochastic process can be read off from the measurement apparatus while the measurement is taking place continuously in time. Let us now check that the observation processes $Y_{t}^{\Lambda}$ and $Y_{t}^{W}$ are indeed self-nondemolition. Let $Z$ be an operator of the form $I \otimes Z \otimes I_{[s}$ on $\mathcal{H} \otimes \mathcal{F}_{s]} \otimes \mathcal{F}_{[s}$. It follows from the quantum Itô rules that for all $t \geq s$

$$
U_{t}^{*} Z U_{t}=U_{s}^{*} Z U_{s}+\int_{s}^{t} U_{r}^{*} \mathcal{L}(Z) U_{r} d r+\int_{s}^{t} U_{r}^{*}[Z, L] U_{r} d A_{r}^{*}+\int_{s}^{t} U_{r}^{*}\left[L^{*}, Z\right] U_{r} d A_{r} .
$$

Since $Z$ is a field operator and $L$ and $H$ are system operators, we have that $\mathcal{L}(Z)=[L, Z]=$ $\left[L^{*}, Z\right]=0$. Therefore we have $U_{t}^{*} Z U_{t}=U_{s}^{*} Z U_{s}$. Using this result with $Z=\Lambda_{s}$, we find

$$
\left[Y_{t}^{\Lambda}, Y_{s}^{\Lambda}\right]=\left[U_{t}^{*} \Lambda_{t} U_{t}, U_{s}^{*} \Lambda_{s} U_{s}\right]=\left[U_{t}^{*} \Lambda_{t} U_{t}, U_{t}^{*} \Lambda_{s} U_{t}\right]=U_{t}^{*}\left[\Lambda_{t}, \Lambda_{s}\right] U_{t}=0,
$$

where we used that $\left[\Lambda_{t}, \Lambda_{s}\right]=0$. In a similar way we find $\left[Y_{t}^{W}, Y_{s}^{W}\right]=0$. That is, both processes $Y^{\Lambda}$ and $Y^{W}$ are self-nondemolition. Note however that $\left[Y_{t}^{\Lambda}, Y_{t}^{W}\right] \neq 0$, we can not observe both processes in a single realization of the experiment.

Define the von Neumann algebra generated by the observations up to time $t$ by $\mathcal{Y}_{t}=\mathrm{vN}\left\{Y_{s} ; 0 \leq\right.$ $s \leq t\}$, where $Y_{s}$ is either $Y_{s}^{\Lambda}$ in case of direct photodetection, or $Y_{s}^{W}$ in case of homodyne detection. We would like to estimate $j_{t}(X)$ for all operators $X \in \mathcal{B}$ based on the observations up to time $t$. That is, we would like to calculate the conditional expectation $\mathbb{P}\left(j_{t}(X) \mid \mathcal{Y}_{t}\right)$ which is the mean least squares estimate of $j_{t}(X)$ given $\mathcal{Y}_{t}$. However, in order for $\mathbb{P}\left(j_{t}(X) \mid \mathcal{Y}_{t}\right)$ to be well-defined, we 
have to show that $j_{t}(X)$ is in the commutant of $\mathcal{Y}_{t}$. This requirement is called the nondemolition property. To show that $j_{t}(X)$ is nondemolished by $\mathcal{Y}_{t}$ we again use that $U_{s}^{*} Z U_{s}=U_{t}^{*} Z U_{t}$ for all operators of the form $I \otimes Z \otimes I_{[s}$ on $\mathcal{H} \otimes \mathcal{F}_{s]} \otimes \mathcal{F}_{[s}$. If we take $Z=A_{s}+A_{s}^{*}$ when $Y_{s}=Y_{s}^{W}$ and $Z=\Lambda_{s}$ when $Y_{s}=Y_{s}^{\Lambda}$, then we obtain

$$
\left[j_{t}(X), Y_{s}\right]=\left[U_{t}^{*} X U_{t}, U_{s}^{*} Z U_{s}\right]=\left[U_{t}^{*} X U_{t}, U_{t}^{*} Z U_{t}\right]=U_{t}^{*}[X, Z] U_{t}=0,
$$

where we used that $[X, Z]=0$ since $X$ is a system operator and $Z$ is a field operator. This establishes the nondemolition property and therefore also that $\mathbb{P}\left(j_{t}(X) \mid \mathcal{Y}_{t}\right)$ is well-defined.

We will now focus on finding the filtering equation, i.e. a recursive equation for updating $\mathbb{P}\left(j_{t}(X) \mid \mathcal{Y}_{t}\right)$ in real time. Note that since $\mathbb{P}\left(j_{t}(X) \mid \mathcal{Y}_{t}\right)$ depends linearly on $X$ and is positive and normalized, we can define an information state $\pi_{t}$ on $\mathcal{B}$ by

$$
\pi_{t}(X)=\iota\left(\mathbb{P}\left(j_{t}(X) \mid \mathcal{Y}_{t}\right)\right)
$$

where $\iota$ maps $Y_{t}$ to a classical process via the spectral theorem. Note that $\pi_{t}$ is a stochastic state, it depends on the observations up to time $t$. The filtering equation propagates the information state $\pi_{t}$ in time and is driven by the observations.

\subsection{Change of state}

\section{Homodyne detection}

Suppose that we are doing a homodyne detection experiment and that the interaction between the system of interest and the field is given by Eq. (18). Our system-observations pair is then given by the quantum probability space $(\mathcal{B} \otimes \mathcal{W}, \mathbb{P})$ and the equations

$$
\begin{aligned}
d j_{t}(X) & =j_{t}(\mathcal{L}(X)) d t+j_{t}\left(\left[L^{*}, X\right]\right) d A_{t}+j_{t}([X, L]) d A_{t}^{*}, \\
d Y_{t} & =j_{t}\left(L+L^{*}\right) d t+d A_{t}+d A_{t}^{*} .
\end{aligned}
$$

Solving the corresponding filtering problem means to find a recursive equation that propagates $\mathbb{P}\left(j_{t}(X) \mid \mathcal{Y}_{t}\right)$ in real time. Our strategy will be to change to a different state $\mathbb{R}^{t}$, the so-called reference state. We will choose the reference state $\mathbb{R}^{t}$ such that the observations have independent increments. That will make it easier to manipulate conditional expectations. Using the Bayes formula we can relate conditional expectations with respect to $\mathbb{R}^{t}$ back to conditional expectations with respect to $\mathbb{P}$.

Note that the process $A_{s}+A_{s}^{*}, 0 \leq s \leq t$ is a Wiener process under $\mathbb{P}$, i.e. it has independent increments under $\mathbb{P}$. Therefore, if we define a reference state

$$
\mathbb{R}^{t}(S)=\mathbb{P}\left(U_{t} S U_{t}^{*}\right), \quad S \in U_{t}^{*} \mathcal{B} \otimes \mathcal{W} U_{t},
$$

then we see that under this state the observations $Y_{s}=j_{s}\left(A_{s}+A_{s}^{*}\right)=j_{t}\left(A_{s}+A_{s}^{*}\right)$ form a Wiener process. That is, under $\mathbb{R}^{t}$, the observation process $Y_{s}=j_{s}\left(A_{s}+A_{s}^{*}\right), 0 \leq s \leq t$ has independent increments. Moreover, under $\mathbb{R}^{t}$ the system $j_{t}(X)(X \in \mathcal{B})$ and the observations $Y_{s}, 0 \leq s \leq t$ are independent. From the definition it is immediate that

$$
\mathbb{P}(S)=\mathbb{R}^{t}\left(U_{t}^{*} S U_{t}\right), \quad S \in \mathcal{B} \otimes \mathcal{W} .
$$


We would now like to use the Bayes formula with $Q=U_{t}$ to relate conditional expectations with respect to $\mathbb{P}$ to conditional expectations with respect to $\mathbb{R}^{t}$. However, $U_{t}$ is not in the commutant of $\mathcal{Y}_{t}$. The following trick, in the spirit of [15], resolves this problem.

We search for an element $Q_{t}$ that satisfies the following two requirements

$$
\begin{aligned}
& \text { 1. } \mathbb{R}^{t}\left(U_{t}^{*} S U_{t}\right)=\mathbb{R}^{t}\left(Q_{t}^{*} S Q_{t}\right), \quad S \in \mathcal{B} \otimes \mathcal{W}, \\
& \text { 2. } Q_{t} \text { is affiliated to the commutant of } \mathcal{Y}_{t} .
\end{aligned}
$$

We could then use the Bayes formula with $Q=Q_{t}$, to obtain

$$
\mathbb{P}\left(j_{t}(X) \mid \mathcal{Y}_{t}\right)=\frac{\mathbb{R}^{t}\left(Q_{t}^{*} j_{t}(X) Q_{t} \mid \mathcal{Y}_{t}\right)}{\mathbb{R}^{t}\left(Q_{t}^{*} Q_{t} \mid \mathcal{Y}_{t}\right)}
$$

The two conditions of Eq. (21) are satisfied if we can find an element $Q_{t}$ that satisfies

1. $\quad Q_{t} U_{t}^{*} v \otimes \Phi=v \otimes \Phi, \quad \forall v \in \mathcal{H}$,

2. $Q_{t}$ is affiliated to the commutant of $\mathcal{Y}_{t}$.

Or equivalently, if we can find an element $V_{t}$ that satisfies

1. $\quad V_{t} v \otimes \Phi=U_{t} v \otimes \Phi, \quad \forall v \in \mathcal{H}$,

2. $V_{t}$ is affiliated to the commutant of $\mathcal{C}_{t}$,

where $\mathcal{C}_{t}=U_{t} \mathcal{Y}_{t} U_{t}^{*}=\mathrm{vN}\left\{A_{s}+A_{s}^{*} ; 0 \leq s \leq t\right\}$. Indeed, if $V_{t}$ satisfies the conditions in Eq. (24), then $Q_{t}=j_{t}\left(V_{t}\right)$ satisfies the conditions in Eq. (23) and subsequently Eq. (21). Let $V_{s}$ for $0 \leq s \leq t$ be given by

$$
d V_{t}=\left\{L\left(d A_{t}^{*}+d A_{t}\right)-\frac{1}{2} L^{*} L d t-i H d t\right\} V_{t}, \quad V_{0}=I,
$$

then $V_{t} v \otimes \Phi=U_{t} v \otimes \Phi$ for all $v \in \mathcal{H}$. Moreover, the equation is driven by $d A_{t}+d A_{t}^{*}$ and the coefficients $L$ and $H$ are in the commutant of $\mathcal{C}_{t}$, i.e. $V_{s}$ is for all $0 \leq s \leq t$ affiliated to the commutant of $\mathcal{C}_{t}$. We conclude that $V_{t}$ given by Eq. (25) satisfies the conditions in Eq. (24).

On $\mathcal{B}$ we define an unnormalized information state by

$$
\sigma_{t}(X)=\mathbb{R}^{t}\left(Q_{t}^{*} j_{t}(X) Q_{t} \mid \mathcal{Y}_{t}\right)=\mathbb{R}^{t}\left(j_{t}\left(V_{t}^{*} X V_{t}\right) \mid \mathcal{Y}_{t}\right) .
$$

From the Bayes rule Eq. (22) it is immediate that

$$
\pi_{t}(X)=\frac{\sigma_{t}(X)}{\sigma_{t}(I)} .
$$

This is the quantum analogue of the classical Kallianpur-Striebel formula. We will now focus on finding an equation that propagates $\sigma_{t}(X)$ in time. From the definition of conditional expectations it easily follows that $\mathbb{R}^{t}\left(j_{t}\left(V_{t}^{*} X V_{t}\right) \mid \mathcal{Y}_{t}\right)=U_{t}^{*} \mathbb{P}\left(V_{t}^{*} X V_{t} \mid \mathcal{C}_{t}\right) U_{t}$. Since $V_{t}$ is driven by classical noise, we can resort to the classical Itô calculus from here onwards. The Itô rule gives

$$
d V_{t}^{*} X V_{t}=V_{t}^{*} \mathcal{L}(X) V_{t} d t+V_{t}^{*}\left(L^{*} X+X L\right) V_{t}\left(d A_{t}+d A_{t}^{*}\right) .
$$


In integral form this reads

$$
V_{t}^{*} X V_{t}=X+\int_{0}^{t} V_{s}^{*} \mathcal{L}(X) V_{s} d s+\int_{0}^{t} V_{s}^{*}\left(L^{*} X+X L\right) V_{s}\left(d A_{s}+d A_{s}^{*}\right) .
$$

Taking the conditional expectation $\mathbb{P}\left(\cdot \mid \mathcal{C}_{t}\right)$ of this equation gives

$$
\mathbb{P}\left(V_{t}^{*} X V_{t} \mid \mathcal{C}_{t}\right)=\mathbb{P}\left(X \mid \mathcal{C}_{t}\right)+\int_{0}^{t} \mathbb{P}\left(V_{s}^{*} \mathcal{L}(X) V_{s} \mid \mathcal{C}_{t}\right) d s+\int_{0}^{t} \mathbb{P}\left(V_{s}^{*}\left(L^{*} X+X L\right) V_{s} \mid \mathcal{C}_{t}\right)\left(d A_{s}+d A_{s}^{*}\right)
$$

where we have approximated the integrals by simple functions in the usual way and have taken the integrators out using the module property of the conditional expectation. For adapted processes $L_{s}$ we have that $\mathbb{P}\left(L_{s} \mid \mathcal{C}_{t}\right)=\mathbb{P}\left(L_{s} \mid \mathcal{C}_{s}\right)$ for all $s \leq t$. This is where our clever choice for the reference state $\mathbb{R}^{t}$ pays off. We use here that $\mathcal{C}_{[s, t]}$ is independent of $\mathcal{C}_{s}$ under $\mathbb{P}$, or equivalently, that $\mathcal{Y}_{[s, t]}$ is independent of $\mathcal{Y}_{s}$ under $\mathbb{R}^{t}$. Note that that is exactly how we had chosen the reference state $\mathbb{R}^{t}$. This means we now have

$$
\mathbb{P}\left(V_{t}^{*} X V_{t} \mid \mathcal{C}_{t}\right)=\mathbb{P}(X)+\int_{0}^{t} \mathbb{P}\left(V_{s}^{*} \mathcal{L}(X) V_{s} \mid \mathcal{C}_{s}\right) d s+\int_{0}^{t} \mathbb{P}\left(V_{s}^{*}\left(L^{*} X+X L\right) V_{s} \mid \mathcal{C}_{s}\right)\left(d A_{s}+d A_{s}^{*}\right) .
$$

Sandwiching with $U_{t}$ leads to the following linear quantum filtering equation

$$
d \sigma_{t}(X)=\sigma_{t}(\mathcal{L}(X)) d t+\sigma_{t}\left(L^{*} X+X L\right) d Y_{t}
$$

This equation is the quantum analogue of the classical Duncan-Mortensen-Zakai equation [20, 12, 29]. Using the Kallianpur-Striebel formula Eq. (26) and the Itô rule, we obtain the normalized quantum filtering equation

$$
d \pi_{t}(X)=\pi_{t}(\mathcal{L}(X)) d t+\left(\pi_{t}\left(L^{*} X+X L\right)-\pi_{t}\left(L^{*}+L\right) \pi_{t}(X)\right)\left(d Y_{t}-\pi_{t}\left(L^{*}+L\right) d t\right) .
$$

This equation is a quantum analogue of the classical Kushner-Stratonovich equation. The process $d Y_{t}-\pi_{t}\left(L^{*}+L\right) d t$ driving the quantum filter is called the innovations or the innovating martingale. In subsection 4.3 we will prove that the innovations indeed form a martingale.

\section{Direct photodetection}

Now suppose that instead of a homodyne detection experiment we are directly counting photons in the field. Our system-observations pair is then given by the quantum probability space $(\mathcal{B} \otimes \mathcal{W}, \mathbb{P})$ and the equations

$$
\begin{aligned}
d j_{t}(X) & =j_{t}(\mathcal{L}(X)) d t+j_{t}\left(\left[L^{*}, X\right]\right) d A_{t}+j_{t}([X, L]) d A_{t}^{*} \\
d Y_{t} & =d \Lambda_{t}+j_{t}(L) d A_{t}^{*}+j_{t}\left(L^{*}\right) d A_{t}+j_{t}\left(L^{*} L\right) d t
\end{aligned}
$$

To solve the corresponding filtering problem, we again change to a different state $\mathbb{R}^{t}$, the so-called reference state for the counting experiment. We choose the reference state such that the observation process has independent increments. Moreover, we want the measure induced on the observations $\mathcal{Y}_{t}$ by the reference measure $\mathbb{R}^{t}$ to be absolutely continuous with respect to the measure induced by $\mathbb{P}$. This last requirement rules out the reference state we used previously for the homodyne case, since under this measure the counting observations will be identical zero, whereas under $\mathbb{P}$ this is not the case. If we would ignore this problem and would proceed naively with the reference state 
from the previous section, we would find that we are unable to find a suitable Radon-Nikodym derivative $Q_{t}$.

We solve the above difficulty by using a reference state that turns the observations into a Poisson process. Let the Weyl operator $W_{t}$ be given by

$$
d W_{t}=\left\{d A_{t}-d A_{t}^{*}-\frac{1}{2} d t\right\} W_{t}, \quad W_{0}=I .
$$

Define $U_{t}^{\prime}=W_{t} U_{t}$, then $U_{t}^{\prime}$ satisfies the following QSDE

$$
d U_{t}^{\prime}=\left\{(L-I) d A_{t}^{*}-\left(L^{*}-I\right) d A_{t}-\frac{1}{2}\left(L^{*} L+I-2 L+2 i H\right) d t\right\}, \quad U_{0}^{\prime}=I .
$$

Let the reference state $\mathbb{R}^{t}$ be given by

$$
\mathbb{R}^{t}(S)=\mathbb{P}\left(U^{\prime}{ }_{t} S U_{t}^{\prime *}\right), \quad S \in U_{t}^{\prime *} \mathcal{B} \otimes \mathcal{W} U_{t}^{\prime}
$$

Note that under $\mathbb{R}^{t}$ we have that the process $Y_{s}, 0 \leq s \leq t$ is a Poisson process, i.e. the observations have independent increments. Moreover, note that under $\mathbb{R}^{t} j_{t}(X)$ and $Y_{s}=j_{s}\left(\Lambda_{s}\right)=j_{t}\left(\Lambda_{s}\right)$ are independent for all $X \in \mathcal{B}$. From the definition it is immediate that

$$
\mathbb{P}(S)=\mathbb{R}^{t}\left(U_{t}^{\prime *} S U_{t}^{\prime}\right), \quad S \in \mathcal{B} \otimes \mathcal{W} .
$$

As before we would now like to apply the Bayes formula. However, Eq. (28) shows that $U_{t}^{\prime}$ is not in the commutant of $\mathcal{Y}_{t}$. We will use the same trick as before to solve this problem [15].

We search for an element $Q_{t}$ that satisfies the following two requirements

1. $\mathbb{R}^{t}\left(U_{t}^{\prime *} S U_{t}^{\prime}\right)=\mathbb{R}^{t}\left(Q_{t}^{*} S Q_{t}\right), \quad S \in \mathcal{B} \otimes \mathcal{W}$,

2. $Q_{t}$ is affiliated to the commutant of $\mathcal{Y}_{t}$.

These conditions are satisfied if we can find an element $Q_{t}$ that satisfies

$$
\begin{aligned}
& \text { 1. } Q_{t} U_{t}^{\prime *} v \otimes \Phi=v \otimes \Phi, \quad \forall v \in \mathcal{H}, \\
& \text { 2. } Q_{t} \text { is affiliated to the commutant of } \mathcal{Y}_{t} .
\end{aligned}
$$

Or equivalently, if we can find an element $V_{t}$ that satisfies

1. $V_{t}^{\prime} v \otimes \Phi=U_{t}^{\prime} v \otimes \Phi, \quad \forall v \in \mathcal{H}$,

2. $V_{t}^{\prime}$ is affiliated to the commutant of $\mathcal{C}_{t}$,

where $\mathcal{C}_{t}=U_{t}^{\prime} \mathcal{Y}_{t} U_{t}^{\prime *}=\operatorname{vN}\left\{W_{s} \Lambda_{s} W_{s}^{*} ; 0 \leq s \leq t\right\}$. Indeed, if we can find a $V_{t}^{\prime}$ satisfying Eq. (31), then $Q_{t}=U_{t}^{\prime *} V_{t}^{\prime} U_{t}^{\prime}$ satisfies Eqs. (30) and (29). Note that $Z_{t}=W_{t} \Lambda_{t} W_{t}^{*}$ is given by

$$
d Z_{t}=d \Lambda_{t}+d A_{t}+d A_{t}^{*}+d t, \quad Z_{0}=0 .
$$

Let $V_{s}^{\prime}$ for $0 \leq s \leq t$ be given by

$$
d V_{t}^{\prime}=\left\{(L-1) d Z_{t}-\frac{1}{2}\left(L^{*} L+I+2 i H\right) d t\right\} V_{t}^{\prime}, \quad V_{0}=I,
$$


then $V_{t}^{\prime} v \otimes \Phi=U_{t}^{\prime} v \otimes \Phi$ for all $v \in \mathcal{H}$. Moreover, the equation is driven by $d Z_{t}$ and the coefficients $L$ and $H$ are in the commutant of $\mathcal{C}_{t}$, i.e. $V_{s}^{\prime}$ is affiliated to the commutant of $\mathcal{C}_{t}$ for all $0 \leq s \leq t$. This means that $V_{t}^{\prime}$ given by Eq. (32) satisfies the conditions in Eq. (31).

We can now apply the Bayes formula with $Q_{t}=U_{t}^{\prime *} V_{t}^{\prime} U_{t}^{\prime}$, i.e.

$$
\mathbb{P}\left(j_{t}(X) \mid \mathcal{Y}_{t}\right)=\frac{\mathbb{R}^{t}\left(Q_{t}^{*} j_{t}(X) Q_{t} \mid \mathcal{Y}_{t}\right)}{\mathbb{R}^{t}\left(Q_{t}^{*} Q_{t} \mid \mathcal{Y}_{t}\right)}, \quad X \in \mathcal{B}
$$

On $\mathcal{B}$ we define an unnormalized information state by

$$
\sigma_{t}(X)=\mathbb{R}^{t}\left(Q_{t}^{*} j_{t}(X) Q_{t} \mid \mathcal{Y}_{t}\right)=\mathbb{R}^{t}\left(U_{t}^{\prime *} V_{t}^{\prime *} X V_{t}^{\prime} U_{t}^{\prime} \mid \mathcal{Y}_{t}\right),
$$

where in the second step we used that $X \in \mathcal{B}$ commutes with $W_{t}$. From the Bayes formula it is immediate that

$$
\pi_{t}(X)=\frac{\sigma_{t}(X)}{\sigma_{t}(I)}, \quad X \in \mathcal{B}
$$

We will now focus on finding an equation that propagates $\sigma_{t}(X)$ in time. From the definition of the conditional expectation it easily follows that $\mathbb{R}^{t}\left(U_{t}^{\prime *} V_{t}^{\prime *} X V_{t}^{\prime} U_{t}^{\prime} \mid \mathcal{Y}_{t}\right)=U_{t}^{\prime *} \mathbb{P}\left(V_{t}^{\prime *} X V_{t}^{\prime} \mid \mathcal{C}_{t}\right) U_{t}^{\prime}$. The Itô rule gives

$$
d V_{t}^{\prime *} X V_{t}^{\prime}=V_{t}^{\prime *} \mathcal{L}(X) V_{t}^{\prime} d t+V_{t}^{\prime}\left(L^{*} X L-X\right) V_{t}^{\prime}\left(d Z_{t}-d t\right)
$$

Using analogous arguments as in the homodyne case, we obtain the linear quantum filter

$$
\sigma_{t}(X)=\sigma_{t}(\mathcal{L}(X)) d t+\left(\sigma_{t}\left(L^{*} X L\right)-\sigma_{t}(X)\right)\left(d Y_{t}-d t\right) .
$$

Using Eq. (33) and the Itô rule, we obtain the normalized quantum filter for photon counting

$$
d \pi_{t}(X)=\pi_{t}(\mathcal{L}(X)) d t+\left(\frac{\pi_{t}\left(L^{*} X L\right)}{\pi_{t}\left(L^{*} L\right)}-\pi_{t}(X)\right)\left(d Y_{t}-\pi_{t}\left(L^{*} L\right) d t\right) .
$$

The process $d Y_{t}-\pi_{t}\left(L^{*} L\right) d t$ is called the innovations or the innovating martingale for the photon counting experiment. In the next subsection we prove that this process indeed forms a martingale.

\subsection{Innovations}

In martingale based approaches to quantum filtering [4],[6], the following theorem is the starting point. The proof below is an adaptation to the Heisenberg picture of the proof in [6]. It can also be found in [8], [7].

Theorem 4.1: Let $Y_{t}$ be given by $Y_{t}=U_{t}^{*} Z_{t} U_{t}$, where $d Z_{t}=a_{t} d \Lambda_{t}+\bar{b}_{t} d A_{t}+b_{t} d A_{t}^{*}, Z_{0}=0$ and $a_{t} \in \mathbb{R}$ and $b_{t} \in \mathbb{C}$ for all $t \geq 0$. Denote by $\mathcal{Y}_{t}$ the von Neumann algebra generated by $Y_{s}$ for $0 \leq s \leq t$. Define the innovations $\tilde{Y}_{t}$ by

$$
\tilde{Y}_{t}=Y_{t}-\int_{0}^{t} \mathbb{P}\left(a_{s} U_{s}^{*} L^{*} L U_{s}+\bar{b}_{s} U_{s}^{*} L U_{s}+b_{s} U_{s}^{*} L^{*} U_{s} \mid \mathcal{Y}_{s}\right) d s .
$$

The innovations are a martingale, i.e. $\mathbb{P}\left(\tilde{Y}_{t} \mid \mathcal{Y}_{s}\right)=\tilde{Y}_{s}$ for all $0 \leq s \leq t$. 
Proof. We have to show that $\mathbb{P}\left(\tilde{Y}_{t}-\tilde{Y}_{s} \mid \mathcal{Y}_{s}\right)=0$ for all $0 \leq s \leq t$. This means we need to prove that $\mathbb{P}\left(\left(\tilde{Y}_{t}-\tilde{Y}_{s}\right) K\right)=0$ for all $0 \leq s \leq t$ and $K \in \mathcal{Y}_{s}$. This is equivalent to showing that

$$
\mathbb{P}\left(Y_{t} K\right)-\mathbb{P}\left(Y_{s} K\right)=\int_{s}^{t} \mathbb{P}\left(\mathbb{P}\left(a_{r} U_{r}^{*} L^{*} L U_{r}+\bar{b}_{r} U_{r}^{*} L U_{r}+b_{r} U_{r}^{*} L^{*} U_{r} \mid \mathcal{Y}_{r}\right) K\right) d r,
$$

for all $0 \leq s \leq t$ and for all $K \in \mathcal{Y}_{s}$. For $t=s$ the above equation is obviously true. It therefore remains to show that for all $K \in \mathcal{Y}_{s}$ and all $s \leq r \leq t$

$$
\begin{aligned}
d \mathbb{P}\left(Y_{r} K\right) & =\mathbb{P}\left(\mathbb{P}\left(a_{r} U_{r}^{*} L^{*} L U_{r}+\bar{b}_{r} U_{r}^{*} L U_{r}+b_{r} U_{r}^{*} L^{*} U_{r} \mid \mathcal{Y}_{r}\right) K\right) d r \\
& =\mathbb{P}\left(a_{r} U_{r}^{*} L^{*} L U_{r} K+\bar{b}_{r} U_{r}^{*} L U_{r} K+b_{r} U_{r}^{*} L^{*} U_{r} K\right) d r
\end{aligned}
$$

This is just a simple exercise in applying the quantum Itô rule and the relation $Y_{r}=U_{r}^{*} Z_{r} U_{r}$.

Acknowledgment. I would like to thank Martin Lindsay and Uwe Franz for a critical reading of a first version of this text.

\section{References}

[1] L. Accardi, A. Frigerio, and Y. Lu. The weak coupling limit as a quantum functional central limit. Commun. Math. Phys., 131:537-570, 1990.

[2] A. Barchielli. Direct and heterodyne detection and other applications of quantum stochastic calculus to quantum optics. Quantum Opt., 2:423-441, 1990.

[3] V. P. Belavkin. Nondemolition stochastic calculus in Fock space and nonlinear filtering and control in quantum systems. In R. Guelerak and W. Karwowski, editors, Proceedings XXIV Karpacz winter school, Stochastic methods in mathematics and physics, pages 310-324. World Scientific, Singapore, 1988.

[4] V. P. Belavkin. Quantum stochastic calculus and quantum nonlinear filtering. J. Multivar. Anal., 42:171-201, 1992.

[5] L. Bouten, J. Stockton, G. Sarma, and H. Mabuchi. Scattering of polarized laser light by an atomic gas in free space: a quantum stochastic differential equation approach. Phys. Rev. A, 75:052111, 2007.

[6] L. M. Bouten, M. I. Guţă, and H. Maassen. Stochastic Schrödinger equations. J. Phys. A, $37: 3189-3209,2004$.

[7] L. M. Bouten and R. Van Handel. On the separation principle of quantum control, 2005. math-ph/0511021.

[8] L. M. Bouten and R. Van Handel. Quantum filtering: a reference probability approach, 2005. math-ph/0508006.

[9] L. M. Bouten, R. Van Handel, and M. James. An introduction to quantum filtering, 2006. math.OC/0601741, to appear in SIAM J. Control Optim. 
[10] C. Cohen Tannoudji, J. Dupont Roc, and G. Grynberg. Photons and Atoms: Introduction to Quantum Electrodynamics. Wiley, 1989.

[11] J. Derezinski and W. De Roeck. Extended weak coupling limit for Pauli-Fierz operators, 2006. math-ph/0610054.

[12] T. Duncan. Evaluation of likelihood functions. Information and Control, (13):62-74, 1968.

[13] F. Fagnola. On quantum stochastic differential equations with unbounded coefficients. Probab. Th. Rel. Fields, 86:501-516, 1990.

[14] J. Gough. Quantum flows as Markovian limit of emission, absorption and scattering interactions. Commun. Math. Phys., 254:489-512, 2005.

[15] A. Holevo. Quantum stochastic calculus. J. Soviet Math., 56:2609-2624, 1991. Translation of Itogi Nauki i Tekhniki, ser. sovr. prob. mat. 36, 3-28, 1990.

[16] R. L. Hudson and K. R. Parthasarathy. Quantum Itô's formula and stochastic evolutions. Commun. Math. Phys., 93:301-323, 1984.

[17] R. V. Kadison and J. R. Ringrose. Fundamentals of the Theory of Operator Algebras, volume I. Academic Press, San Diego, 1983.

[18] G. Lindblad. On the generators of quantum dynamical semigroups. Commun. Math. Phys., 48:119-130, 1976.

[19] H. Maassen. Quantum probability applied to the damped harmonic oscillator. In S. Attal and J. Lindsay, editors, Quantum Probability Communications, volume XII, pages 23-58. World Scientific, Singapore, 2003.

[20] R. Mortensen. Optimal Control of Continuous-Time Stochastic Systems. PhD thesis, Univ. California, Berkeley, 1966.

[21] K. R. Parthasarathy. An Introduction to Quantum Stochastic Calculus. Birkhäuser, Basel, 1992.

[22] D. Petz. An Invitation to the Algebra of Canonical Commutation Relations. Leuven University Press, Leuven, 1990.

[23] M. Reed and B. Simon. Functional Analysis, volume 1 of Methods of Modern Mathematical Physics. Elsevier, 1980.

[24] I. Segal. Tensor algebras over Hilbert spaces. I. Trans. Am. Math. Soc., 81:106-134, 1956.

[25] J. K. Stockton. Continuous quantum measurement of cold alkali-atom spins. PhD thesis, California Institute of Technology, 2006.

[26] M. Takesaki. Conditional expectations in von Neumann algebras. J. Funct. Anal., 9:306-321, 1971.

[27] J. von Neumann. Zur Algebra der Funktionaloperationen und Theorie der normalen Operatoren. Math. Ann., 102:370-427, 1929.

[28] D. Williams. Probability with Martingales. Cambridge University Press, Cambridge, 1991.

[29] M. Zakai. On the optimal filtering of diffusion processes. Z. Wahrsch. Verw. Geb., 11:230-243, 1969. 